\title{
GENERALIZED GREEN FUNCTIONS AND UNIPOTENT CLASSES FOR FINITE REDUCTIVE GROUPS, II
}

\author{
TOSHIAKI SHOJI
}

\begin{abstract}
This paper is concerned with the problem of the determination of unknown scalars involved in the algorithm of computing the generalized Green functions of reductive groups $G$ over a finite field. In the previous paper, we have treated the case where $G=S L_{n}$. In this paper, we determine the scalars in the case where $G$ is a classical group $S p_{2 n}$ or $S O_{N}$ for arbitrary characteristic.
\end{abstract}

\section{§0. Introduction}

This paper is a sequel to [S2]. Our aim is to remove an ambiguity from the algorithm of computing generalized Green functions of reductive groups due to Lusztig. Let $G$ be a connected reductive group defined over a finite field $\mathbf{F}_{q}$ with Frobenius map $F$. Let $p$ be the characteristic of $\mathbf{F}_{q}$. In [S2], we have treated the case where $G=S L_{n}$. In this paper we consider the case where $G=S p_{2 n}$ or $S O_{N}$ for arbitrary $p$. The case where $G=\operatorname{Spin}_{N}$ will be treated in a separate paper.

In [S1] it was shown, in the case of $S p_{2 n}$ or $S O_{N}$ with $p \neq 2$, that there exists a representative in $C^{F}$ for each unipotent class $C$, called a distinguished element there (in this paper we call it a split element) which behaves well with respect to the computation of Green functions. Our result in this paper shows that the split elements behave well for any type of generalized Green functions. We also show, in the case where $p=2$, that such a good representative (called a split element) exists for $G=S p_{2 n}$ or $S O_{2 n}$. This was not known even for the case of Green functions.

The main ingredient for the proof is a variant of the restriction theorem ([L1]) for the generalized Springer correspondence. The restriction theorem is a powerful tool for determining the generalized Springer correspondence, and it was used in [LS], [Sp2] quite effectively. We extend this theorem so that it involves the information on the Frobenius action. In [S2], we have

Received December 21, 2006.

2000 Mathematics Subject Classification: 20G05, 20G40. 
obtained the results by investigating the Frobenius action on the cohomology group $H_{c}^{a_{0}+r}\left(\mathcal{P}_{u}, \dot{\mathcal{E}}\right)$. But this requires a precise information on the geometry of $\mathcal{P}_{u}$ related to the local system $\dot{\mathcal{E}}$. In the case of classical groups, one can avoid to deal with $\mathcal{P}_{u}$ by considering the restriction theorem as above.

\section{$\S 1$. A variant of the restriction theorem}

1.1. We follow the notation in Section 1 in [S2]. In particular, $G$ is a connected reductive group over a finite field $\mathbf{F}_{q}$, with Frobenius map $F$. Let $k$ be an algebraic closure of $\mathbf{F}_{q}$ and $p$ the characteristic of $k$. Let $P=L U_{P}$ be a parabolic subgroup of $G$, where $L$ is a Levi subgroup of $P$ and $U_{P}$ is the unipotent radical of $P$, and let $\mathcal{E}$ be a cuspidal local system on a unipotent class $C$ in $L$. As in (1.2.2) in [S2], one can define a perverse sheaf $K$ on $G$ associated to the triple $(L, C, \mathcal{E})$. Then $K$ is a semisimple perverse sheaf with End $K \simeq \overline{\mathbf{Q}}_{l}[\mathcal{W}]$, where $\mathcal{W}=N_{G}(L) / L$ is a Coxeter group. Thus $K$ is decomposed as

$$
K=\bigoplus_{E \in \mathcal{W}^{\wedge}} V_{E} \otimes K_{E}
$$

where $K_{E}$ is a simple perverse sheaf on $G$ such that $V_{E}=\operatorname{Hom}\left(K_{E}, K\right)$ is an irreducible $\mathcal{W}$-module corresponding to $E \in \mathcal{W}^{\wedge}$. Put $d=\operatorname{dim} Z_{L}^{0}$, where $Z_{L}$ is the center of $L$. Let $G_{\text {uni }}$ be the unipotent variety of $G$, and $\mathcal{N}_{G}$ the set of all the pairs $\left(C^{\prime}, \mathcal{E}^{\prime}\right)$, where $C^{\prime}$ is a unipotent class in $G$ and $\mathcal{E}^{\prime}$ is a $G$-equivariant simple local system on $C^{\prime}$. Then it is known that $\left.K[-d]\right|_{G_{\text {uni }}}$ is a semisimple perverse sheaf on $G_{\text {uni }}$, and it is decomposed as

$$
\left.K[-d]\right|_{G_{\mathrm{uni}}} \simeq \bigoplus_{\left(C^{\prime}, \mathcal{E}^{\prime}\right) \in \mathcal{N}_{G}} V_{\left(C^{\prime}, \mathcal{E}^{\prime}\right)} \otimes \operatorname{IC}\left(\bar{C}^{\prime}, \mathcal{E}^{\prime}\right)\left[\operatorname{dim} C^{\prime}\right],
$$

where $V_{\left(C^{\prime}, \mathcal{E}^{\prime}\right)}$ is the multiplicity space for the simple perverse sheaf $\operatorname{IC}\left(\bar{C}^{\prime}, \mathcal{E}^{\prime}\right)\left[\operatorname{dim} C^{\prime}\right]$ on $G_{\text {uni }}$ (cf. [S2, (1.2.4)]). Thus $\left.K_{E}\right|_{G_{\text {uni }}}$ coincides with some $\operatorname{IC}\left(\bar{C}^{\prime}, \mathcal{E}^{\prime}\right)$ up to shift, and $V_{\left(C^{\prime}, \mathcal{E}^{\prime}\right)}$ coincides with $V_{E}$. It turns out that all the irreducible $\mathcal{W}$-modules are realized as $V_{\left(C^{\prime}, \mathcal{E}^{\prime}\right)}$ for some pair $\left(C^{\prime}, \mathcal{E}^{\prime}\right)$. Thus we have an injective map $\mathcal{W}^{\wedge} \rightarrow \mathcal{N}_{G}$ by $E=V_{\left(C^{\prime}, \mathcal{E}^{\prime}\right)} \rightarrow\left(C^{\prime}, \mathcal{E}^{\prime}\right)$, whose image we denote by $\mathcal{N}_{G}(C, \mathcal{E})$. Let $\mathcal{M}_{G}$ be the set of triples $(L, C, \mathcal{E})$ up to $G$-conjugacy, where $L$ is a Levi subgroup of a parabolic subgroup of $G$ and $\mathcal{E}$ is a cuspidal local system on a unipotent class $C$ of $L$. The above injective maps form a bijection

$$
\coprod_{(L, C, \mathcal{E}) \in \mathcal{M}_{G}}\left(N_{G}(L) / L\right)^{\wedge} \longrightarrow \mathcal{N}_{G}
$$


which is the so-called generalized Springer correspondence ([L1, 6.5]).

1.2. Let $Q \supset P$ be a parabolic subgroup of $G$ with the Levi subgroup $M$ such that $M \supset L$. Then $\mathcal{W}_{1}=N_{M}(L) / L$ is in a natural way a subgroup of $\mathcal{W}$. Replacing $G$ by $M$, we have a subset $\mathcal{N}_{M}(C, \mathcal{E})$ of $\mathcal{N}_{M}$. For each $\left(C^{\prime}, \mathcal{E}^{\prime}\right) \in \mathcal{N}_{G}(C, \mathcal{E})\left(\operatorname{resp} .\left(C_{1}, \mathcal{E}_{1}\right) \in \mathcal{N}_{M}(C, \mathcal{E})\right)$, we denote by $E$ (resp. $\left.E_{1}\right)$ the corresponding irreducible representation of $\mathcal{W}$ (resp. $\mathcal{W}_{1}$ ) under (1.1.3).

Let $\pi_{Q}: Q \rightarrow M$ be the natural projection. Assume that $\left(C_{1}, \mathcal{E}_{1}\right) \in$ $\mathcal{N}_{M}(C, \mathcal{E})$, and that $\left(C^{\prime}, \mathcal{E}^{\prime}\right) \in \mathcal{N}_{G}$. We denote by $f_{C_{1}, C^{\prime}}: C_{1} U_{Q} \cap C^{\prime} \rightarrow C_{1}$ the restriction of $\pi_{Q}$. Then $\mathcal{F}=R^{2 d_{C_{1}, C^{\prime}}}\left(f_{C_{1}, C^{\prime}}\right) ! \mathcal{E}^{\prime}$ is a semisimple $M$ equivariant local system on $C_{1}$, where $d_{C_{1}, C^{\prime}}=\left(\operatorname{dim} C^{\prime}-\operatorname{dim} C_{1}\right) / 2$. We define an integer $m_{\mathcal{E}_{1}, \mathcal{E}^{\prime}}$ to be the multiplicity of $\mathcal{E}_{1}$ in $\mathcal{F}$. Lusztig proved the following restriction theorem on the generalized Springer correspondence.

Theorem 1.3. (Lusztig [L1, Theorem 8.3]) Under the above setting, $\left(C^{\prime}, \mathcal{E}^{\prime}\right) \in \mathcal{N}_{G}(C, \mathcal{E})$ if and only if $m_{\mathcal{E}_{1}, \mathcal{E}^{\prime}} \neq 0$. Moreover in that case we have

$$
m_{\mathcal{E}_{1}, \mathcal{E}^{\prime}}=\left\langle\operatorname{Res} E, E_{1}\right\rangle_{\mathcal{W}_{1}},
$$

where $\langle,\rangle_{\mathcal{W}_{1}}$ is the inner product of two representations of $\mathcal{W}_{1}$ (regarded as characters), and $\operatorname{Res} E$ is the restriction of $E$ on $\mathcal{W}_{1}$.

1.4. Let $u \in C^{\prime}$ and $v \in C_{1}$, and consider the component group $A_{G}(u)$ and $A_{M}(v)$. The set of $G$-equivariant simple local systems on $C^{\prime}$ is in 1:1 correspondence with the set $A_{G}(u)^{\wedge}$ of irreducible characters of $A_{G}(u)$, and a similar fact holds also for $M$. As described in [LS], the integer $m_{\mathcal{E}_{1}, \mathcal{E}^{\prime}}$ can be interpreted in terms of the representations of $A_{G}(u)$ and $A_{M}(v)$, which we explain below. Let $\mathcal{F}_{v}$ be the stalk of $\mathcal{F}$ at $v \in C_{1}$. Then we have

$$
\mathcal{F}_{v} \simeq H_{c}^{2 d_{C_{1}, C^{\prime}}}\left(C^{\prime} \cap v U_{Q}, \mathcal{E}^{\prime}\right) .
$$

Let $\pi: \widetilde{C}^{\prime}=Z_{G}^{0}(u) \backslash G \rightarrow C^{\prime}, Z_{G}^{0}(u) g \mapsto g^{-1} u g$ be the finite covering of $C^{\prime}$ with group $A_{G}(u)$. Let $X=\left(C^{\prime} \cap v U_{Q}\right) \times_{C^{\prime}} \widetilde{C}^{\prime}$ be the fibre product of $C^{\prime} \cap v U_{Q}$ with $\widetilde{C}^{\prime}$ over $C^{\prime}$, and let $\tilde{\pi}: X \rightarrow C^{\prime} \cap v U_{Q}$ be the base change of $\pi$. Then we have

$$
H_{c}^{2 d_{C_{1}, C^{\prime}}}\left(C^{\prime} \cap v U_{Q}, \widetilde{\pi}_{*} \overline{\mathbf{Q}}_{l}\right) \simeq H_{c}^{2 d_{C_{1}, C^{\prime}}}\left(X, \overline{\mathbf{Q}}_{l}\right),
$$

and $A_{G}(u)$ acts naturally on the right hand side. Now $\widetilde{\pi}_{*} \overline{\mathbf{Q}}_{l}$ can be decomposed as $\widetilde{\pi}_{*} \overline{\mathbf{Q}}_{l}=\sum_{\rho} V_{\rho} \otimes \mathcal{E}_{\rho}$, where $\rho$ runs over all the irreducible 
characters of $A_{G}(u)$. Here $\mathcal{E}_{\rho}$ is the $G$-equivariant simple local system on $C^{\prime}$ corresponding to $\rho$ and $V_{\rho}$ is the corresponding irreducible representation of $A_{G}(u)$. It follows that

$$
H_{c}^{2 d_{C_{1}, C^{\prime}}}\left(C^{\prime} \cap v U_{Q}, \mathcal{E}_{\rho}\right) \simeq\left(H_{c}^{2 d_{C_{1}, C^{\prime}}}\left(X, \overline{\mathbf{Q}}_{l}\right) \otimes V_{\rho^{*}}\right)^{A_{G}(u)},
$$

where $\rho^{*}$ is the dual representation of $\rho$. On the other hand, the semisimple local system $\mathcal{F}$ can be written as $\mathcal{F}=\sum_{\rho_{1}} m_{\rho_{1}} \mathcal{E}_{\rho_{1}}$, where $\mathcal{E}_{\rho_{1}}$ is the irreducible local system on $C_{1}$ corresponding to $\rho_{1} \in A_{M}(v)^{\wedge}$ and $m_{\rho_{1}}$ is the multiplicity of $\mathcal{E}_{\rho_{1}}$ in $\mathcal{F}$. By taking the stalk at $v$, we have $\mathcal{F}_{v}=$ $\sum_{\rho_{1}} m_{\rho_{1}}\left(\mathcal{E}_{\rho_{1}}\right)_{v}$. Here $\left(\mathcal{E}_{\rho_{1}}\right)_{v}$ is an irreducible $A_{M}(v)$-module corresponding to $\rho_{1}$. Note that if $\mathcal{E}^{\prime}=\mathcal{E}_{\rho}$, and $\mathcal{E}_{1}=\mathcal{E}_{\rho_{1}}$, we have $m_{\mathcal{E}_{1}, \mathcal{E}^{\prime}}=m_{\rho_{1}}$. Now $Z_{M}(v)$ acts on $C^{\prime} \cap v U_{Q}$ by conjugation, and it induces an action of $A_{M}(v)$ on $H_{c}^{2 d_{C_{1}, C^{\prime}}}\left(C^{\prime} \cap v U_{Q}, \mathcal{E}^{\prime}\right)$. We have

$$
\begin{aligned}
m_{\mathcal{E}_{1}, \mathcal{E}^{\prime}} & =\left\langle H_{c}^{2 d_{C_{1}, C^{\prime}}}\left(C^{\prime} \cap v U_{Q}, \mathcal{E}_{\rho}\right), \rho_{1}\right\rangle_{A_{M}(v)} \\
& \left.=\left\langle H_{c}^{2 d_{C_{1}, C^{\prime}}}\left(X, \overline{\mathbf{Q}}_{l}\right) \otimes V_{\rho^{*}}\right)^{A_{G}(u)}, \rho_{1}\right\rangle_{A_{M}(v)},
\end{aligned}
$$

where $\langle,\rangle_{A_{M}(v)}$ denotes the inner product of characters of $A_{M}(v)$.

By Proposition 1.2 in [L1], it is known that $\operatorname{dim} X \leq d_{C_{1}, C^{\prime}}$. Thus $H_{c}^{2 d_{C_{1}, C^{\prime}}}\left(X, \overline{\mathbf{Q}}_{l}\right)$ has a basis corresponding to the set of irreducible components of $X$ of dimension $d_{C_{1}, C^{\prime}}$, and the action of $A_{G}(u)$ on $H_{C}^{2 d_{C_{1}, C^{\prime}}}\left(X, \overline{\mathbf{Q}}_{l}\right)$ coincides with the permutation action of $A_{G}(u)$ on those irreducible components of $X$. Since $\widetilde{C}^{\prime}=Z_{G}^{0}(u) \backslash G$, we have

$$
\begin{aligned}
X & =\left\{\left(y, Z_{G}^{0}(u) g\right) \in\left(C^{\prime} \cap v U_{Q}\right) \times \widetilde{C}^{\prime} \mid y=g^{-1} u g\right\} \\
& =\left\{Z_{G}^{0}(u) g \mid g^{-1} u g \in v U_{Q}\right\} \\
& =Z_{G}^{0}(u) \backslash\left\{g \in G \mid g^{-1} u g \in v U_{Q}\right\} .
\end{aligned}
$$

Put $Y_{u, v}=\left\{g \in G \mid g^{-1} u g \in v U_{Q}\right\}$. Then $Z_{G}(u) \times Z_{M}(v)$ acts on $Y_{u, v}$ by $\left(z, z^{\prime}\right): g \mapsto z g z^{\prime-1}$ for $z \in Z_{G}(u), z^{\prime} \in Z_{M}(v)$, and the projection $Y_{u, v} \rightarrow X=Z_{G}^{0}(u) \backslash Y_{u, v}$ gives a bijection between the set of irreducible components of $X$ and $Y_{u, v}$, which is compatible with the action of $A_{G}(u)$ and $A_{M}(v)$. Note that

$$
\begin{aligned}
\operatorname{dim} Y_{u, v} & =\operatorname{dim} X+\operatorname{dim} Z_{G}^{0}(u) \\
& =d_{C_{1}, C^{\prime}}+\operatorname{dim} Z_{G}^{0}(u) \\
& =\left(\operatorname{dim} Z_{G}(u)+\operatorname{dim} Z_{M}(v)\right) / 2+\operatorname{dim} U_{Q} .
\end{aligned}
$$


Let $X_{u, v}$ be the set of irreducible components of $Y_{u, v}$ of dimension $d_{C_{1}, C^{\prime}}+$ $\operatorname{dim} Z_{G}^{0}(u)$. It follows from the above discussion, we have

Corollary 1.5. (Lusztig-Spaltenstein [LS, 0.4, (4)]) Let $\varepsilon_{u, v}$ be the permutation representation of $A_{G}(u) \times A_{M}(v)$ on $X_{u, v}$. Then we have

$$
\left\langle\operatorname{Res} E, E_{1}\right\rangle_{\mathcal{W}_{1}}=m_{\mathcal{E}_{1}, \mathcal{E}^{\prime}}=\left\langle\varepsilon_{u, v}, \rho \otimes \rho_{1}^{*}\right\rangle_{A_{G}(u) \times A_{M}(v)} .
$$

1.6. We want to consider a variant of Corollary 1.5 which involves the Frobenius action. Assume that $P$ is $F$-stable, and that the triple $(L, C, \mathcal{E}) \in \mathcal{M}_{G}$ is $F$-stable. We choose $u_{0} \in C^{F}$ and fix an isomorphism $\varphi_{0}: F^{*} \mathcal{E} \stackrel{\sim}{\rightarrow} \mathcal{E}$ so that the induced isomorphism $\mathcal{E}_{u_{0}} \rightarrow \mathcal{E}_{u_{0}}$ is of finite order. $\varphi_{0}$ induces an isomorphism $\varphi: F^{*} K \stackrel{\sim}{\rightarrow} K$. For each pair $\left(C^{\prime}, \mathcal{E}^{\prime}\right) \in \mathcal{N}_{G}^{F}$, we choose $u \in C^{\prime F}$. We fix an isomorphism $\psi_{\mathcal{E}^{\prime}}: F^{*} \mathcal{E}^{\prime} \stackrel{\sim}{\rightarrow} \mathcal{E}^{\prime}$ as follows; $F$ acts naturally on $A_{G}(u)$, and we consider the semidirect product $\widetilde{A}_{G}(u)=\langle\tau\rangle \ltimes A_{G}(u)$, where $\tau$ is the restriction of $F$ on $A_{G}(u)$. Since $\left(C^{\prime}, \mathcal{E}^{\prime}\right)$ is $F$-stable, $\rho$ is $F$-stable. We choose an extension $\widetilde{\rho}$ of $\rho$ to $\widetilde{A}_{G}(u)$ and fix an isomorphism $\psi_{\mathcal{E}^{\prime}}$ so that the induced isomorphism $\mathcal{E}_{u}^{\prime} \rightarrow \mathcal{E}_{u}^{\prime}$ corresponds to the action of $\tau$ on $\widetilde{\rho}$. Now $\psi_{\mathcal{E}^{\prime}}$ induces an isomorphism $\widetilde{\psi}_{\mathcal{E}^{\prime}}: F^{*} \operatorname{IC}\left(\bar{C}^{\prime}, \mathcal{E}^{\prime}\right)\left[\operatorname{dim} C^{\prime}\right] \stackrel{\sim}{\rightarrow} \operatorname{IC}\left(\bar{C}^{\prime}, \mathcal{E}^{\prime}\right)\left[\operatorname{dim} C^{\prime}\right]$. The isomorphism $\varphi$ also induces an isomorphism $\left.\left.F^{*} K[-d]\right|_{G_{\text {uni }}} \stackrel{\sim}{\rightarrow} K[-d]\right|_{G_{\text {uni }}}$, which we also denote by $\varphi$. Then under the decomposition of (1.1.2), $\varphi$ induces an isomorphism

$$
V_{\left(C^{\prime} \mathcal{E}^{\prime}\right)} \otimes F^{*} \operatorname{IC}\left(\bar{C}^{\prime}, \mathcal{E}^{\prime}\right)\left[\operatorname{dim} C^{\prime}\right] \stackrel{\sim}{\longrightarrow} V_{\left(C^{\prime} \mathcal{E}^{\prime}\right)} \otimes \operatorname{IC}\left(\bar{C}^{\prime}, \mathcal{E}^{\prime}\right)\left[\operatorname{dim} C^{\prime}\right]
$$

for each pair $\left(C^{\prime}, \mathcal{E}^{\prime}\right) \in \mathcal{N}_{G}^{F}$, and one can define a linear isomorphism $\sigma_{\left(C^{\prime}, \mathcal{E}^{\prime}\right)}$ on $V_{\left(C^{\prime}, \mathcal{E}^{\prime}\right)}$ such that this isomorphism can be written as $\sigma_{\left(C^{\prime}, \mathcal{E}^{\prime}\right)} \otimes \widetilde{\psi}_{\mathcal{E}^{\prime}}$. Now $F$ acts naturally on $\mathcal{W}=N_{G}(L) / L$, and $\sigma_{\left(C^{\prime}, \mathcal{E}^{\prime}\right)}$ becomes $\mathcal{W}$-semilinear, namely we have a relation $\sigma_{\left(C^{\prime}, \mathcal{E}^{\prime}\right)} w=F(w) \sigma_{\left(C^{\prime}, \mathcal{E}^{\prime}\right)}$ on $V_{\left(C^{\prime}, \mathcal{E}^{\prime}\right)}$ for each $w \in$ $\mathcal{W}$. Replacing $G$ by $M$, we can define a $\mathcal{W}_{1}$-semilinear map $\sigma_{\left(C_{1}, \mathcal{E}_{1}\right)}$ on $V_{\left(C_{1}, \mathcal{E}_{1}\right)}$ for each pair $\left(C_{1}, \mathcal{E}_{1}\right) \in \mathcal{N}_{M}^{F}$. The irreducible $\mathcal{W}$-module $V_{\left(C^{\prime}, \mathcal{E}^{\prime}\right)}$ can be written as a $\mathcal{W}_{1}$-module

$$
V_{\left(C^{\prime}, \mathcal{E}^{\prime}\right)}=\sum_{E_{1} \in \mathcal{W}_{1}^{\wedge}} M_{E_{1}} \otimes E_{1}
$$

where $M_{E_{1}}$ is the multiplicity space of the irreducible $\mathcal{W}_{1}$-module $E_{1}$ and is realized as $M_{E_{1}}=\operatorname{Hom}_{\mathcal{W}_{1}}\left(E_{1}, V_{\left(C^{\prime}, \mathcal{E}^{\prime}\right)}\right)$. Suppose that $E_{1} \simeq V_{\left(C_{1}, \mathcal{E}_{1}\right)}$ under the generalized Springer correspondence for $M$. If $E_{1}$ is $F$-stable, $\left(C_{1}, \mathcal{E}_{1}\right) \in \mathcal{N}_{M}^{F}$, and we have an isomorphism $\sigma_{\left(C_{1}, \mathcal{E}_{1}\right)}$ on $E_{1}$. One can define 
a map $\sigma_{\mathcal{E}_{1}, \mathcal{E}^{\prime}}: M_{E_{1}} \rightarrow M_{E_{1}}$ by $f \mapsto \sigma_{\left(C^{\prime}, \mathcal{E}^{\prime}\right)} \circ f \circ \sigma_{\left(C_{1}, \mathcal{E}_{1}\right)}^{-1}$. The linear map $\sigma_{\left(C^{\prime}, \mathcal{E}^{\prime}\right)}$ stabilizes the subspace $M_{E_{1}} \otimes E_{1}$ and we have

$$
\left.\sigma_{\left(C^{\prime}, \mathcal{E}^{\prime}\right)}\right|_{M_{E_{1}} \otimes E_{1}}=\sigma_{\mathcal{E}_{1}, \mathcal{E}^{\prime}} \otimes \sigma_{\left(C_{1}, \mathcal{E}_{1}\right)} .
$$

On the other hand, since $F\left(C^{\prime}\right)=C^{\prime}, F\left(C_{1}\right)=C_{1}$, the map $f_{C_{1}, C^{\prime}}$ is $F$-equivariant. Hence $\psi_{\mathcal{E}^{\prime}}: F^{*} \mathcal{E}^{\prime} \stackrel{\sim}{\rightarrow} \mathcal{E}^{\prime}$ induces an isomorphism $\psi_{C_{1}, C^{\prime}}$ : $F^{*} \mathcal{F} \stackrel{\sim}{\longrightarrow}$, and so a linear isomorphism $\mathcal{F}_{v} \rightarrow \mathcal{F}_{v}$ which we denote by the same symbol $\psi_{C_{1}, C^{\prime}}$. Now the local system $\mathcal{F}$ on $C_{1}$ corresponds to a representation $V$ of $A_{M}(v)$. $V$ can be decomposed as

$$
V=\sum_{\rho_{1} \in A_{M}(v)^{\wedge}} M_{\rho_{1}} \otimes \rho_{1},
$$

where $M_{\rho_{1}}=\operatorname{Hom}_{A_{M}(v)}\left(\rho_{1}, V\right)$ is the multiplicity space of the irreducible representation $\rho_{1}$. $F$ acts on $A_{M}(v)$, and as in the case of $G$ we consider the semidirect product $\widetilde{A}_{M}(v)=\langle\tau\rangle \ltimes A_{M}(v)$, where $\tau$ is the restriction of $F$ on $A_{M}(v)$. For each $\left(C_{1}, \mathcal{E}_{1}\right) \in \mathcal{N}_{M}^{F}$, we fix an isomorphism $\psi_{\mathcal{E}_{1}}: F^{*} \mathcal{E}_{1} \stackrel{\sim}{\rightarrow} \mathcal{E}_{1}$ as in $G$ by using an extension $\widetilde{\rho}_{1}$ of $\rho_{1}$ to $\widetilde{A}_{M}(v)$. Now $\psi_{C_{1}, C^{\prime}}$ stabilizes the subspace $M_{\rho_{1}} \otimes \rho_{1}$ for an $F$-stable $\rho_{1} \in A_{M}(v)^{\wedge}$, and as in (1.6.2) one can define a linear map $\psi_{\rho_{1}, \rho}$ on $M_{\rho_{1}}$ such that

$$
\left.\psi_{C_{1}, C^{\prime}}\right|_{M_{\rho_{1}} \otimes \rho_{1}}=\psi_{\rho_{1}, \rho} \otimes \psi_{\mathcal{E}_{1}} .
$$

The following result gives an $F$-twisted version of the restriction theorem (Theorem 1.3). The proof is done by chasing the argument in [L1].

Proposition 1.7. Under the notation as above, we have

$$
\operatorname{Tr}\left(\sigma_{\mathcal{E}_{1}, \mathcal{E}^{\prime}}, M_{E_{1}}\right)=q^{-d_{C_{1}, C^{\prime}}+\operatorname{dim} U_{Q}} \operatorname{Tr}\left(\psi_{\rho_{1}, \rho}, M_{\rho_{1}}\right) .
$$

1.8. Let $Y_{u, v}$ be as in 1.4. Assume that $Q$ is $F$-stable. Since $u, v$ are $F$-stable, $Y_{u, v}$ is $F$-stable, and so $F$ acts as a permutation on $X_{u, v}$. On the other hand, $F$ acts naturally on $A(u, v)=A_{G}(u) \times A_{M}(v)$, and we denote by $\widetilde{A}(u, v)$ the semidirect product group $\langle\tau\rangle \ltimes A(u, v)$. Then the permutation representation $\varepsilon_{u, v}$ is extended to a representation of $\widetilde{A}(u, v)$, which we denote by $\widetilde{\varepsilon}_{u, v}$. Now $\psi_{\mathcal{E}^{\prime}}$ and $\psi_{\mathcal{E}_{1}}$ determine an extension of $\rho \otimes \rho_{1}^{*}$ to $\widetilde{A}(u, v)$, which we denote by $\widetilde{\rho \otimes \rho_{1}^{*}}$. By chasing the argument in 1.4 , we see that

$$
\operatorname{Tr}\left(\psi_{\rho_{1}, \rho}, M_{\rho_{1}}\right)=\left\langle\widetilde{\varepsilon}_{u, v}, \widetilde{\rho \otimes \rho_{1}^{*}}\right\rangle_{A(u, v) \tau},
$$


where in general

$$
\left\langle V_{1}, V_{2}\right\rangle_{A(u, v) \tau}=|A(u, v)|^{-1} \sum_{a \in A(u, v)} \operatorname{Tr}\left(a \tau, V_{1}\right) \operatorname{Tr}\left((a \tau)^{-1}, V_{2}\right)
$$

for representations $V_{1}, V_{2}$ of $\widetilde{A}(u, v)$. Hence combined with Proposition 1.7, we have a variant of Corollary 1.5 involving the Frobenius action.

COROLlary 1.9. Let the notations be as above. Then we have

$$
\operatorname{Tr}\left(\sigma_{\mathcal{E}_{1}, \mathcal{E}^{\prime}}, M_{E_{1}}\right)=q^{-d_{C_{1}, C^{\prime}}+\operatorname{dim} U_{Q}}\left\langle\widetilde{\varepsilon}_{u, v}, \widetilde{\rho \otimes \rho_{1}^{*}}\right\rangle_{A(u, v) \tau} .
$$

1.10. We shall connect the above results to the discussion on generalized Green functions in [S2, Section 1]. Take $j=\left(C^{\prime}, \mathcal{E}^{\prime}\right) \in \mathcal{N}_{G}^{F}$ and let $\psi_{0}=\psi_{\mathcal{E}^{\prime}}: F^{*} \mathcal{E}^{\prime} \stackrel{\sim}{\rightarrow} \mathcal{E}^{\prime}$ be defined in 1.6. $\psi_{0}$ determines the $G^{F_{-}}$ invariant function $Y_{j}^{0}$ on the set $G_{\text {uni }}^{F}$ as in [S2, 1.3]. On the other hand, let $\widetilde{\mathcal{W}}=\mathcal{W} \rtimes\langle c\rangle$ be the semidirect product, where $c$ is a Coxeter group automorphism on $\mathcal{W}$ induced from the action of $F$. In the decomposition in (1.1.1), one can define an isomorphism $\varphi_{E}: F^{*} K_{E} \stackrel{\sim}{\rightarrow} K_{E}$ so that the induced map $\sigma_{E}^{\prime}: V_{E} \rightarrow V_{E}$ makes the irreducible $\mathcal{W}$-module $V_{E}$ the preferred extension to $\widetilde{\mathcal{W}}$ (cf. [L2, IV, (17.2)]). Put

$$
\begin{aligned}
a_{0} & =-\operatorname{dim} Z_{L}^{0}-\operatorname{dim} C^{\prime}, \\
r & =\operatorname{dim} G-\operatorname{dim} L+\operatorname{dim}\left(C \times Z_{L}^{0}\right) .
\end{aligned}
$$

The we have

$$
a_{0}+r=\left(\operatorname{dim} G-\operatorname{dim} C^{\prime}\right)-(\operatorname{dim} L-\operatorname{dim} C) .
$$

We have $\left.\mathcal{H}^{a_{0}}\left(K_{E}\right)\right|_{C^{\prime}}=\mathcal{E}^{\prime}$ and we define $\psi: F^{*} \mathcal{E}^{\prime} \stackrel{\sim}{\rightarrow} \mathcal{E}^{\prime}$ so that $q^{\left(a_{0}+r\right) / 2} \psi$ coincides with the map defined by $\varphi_{E}: F^{*} \mathcal{H}^{a_{0}}\left(K_{E}\right) \stackrel{\sim}{\rightarrow} K_{E}$. The function $Y_{j}$ is defined as the characteristic function of $\mathcal{E}^{\prime}$ through $\psi$, extended by 0 to the function on $G_{\text {uni }}^{F}$ (see $[\mathrm{S} 2,1.3]$ ). Since $\mathcal{E}^{\prime}$ is a simple local system, there exists $\gamma \in \overline{\mathbf{Q}}_{l}^{*}$ such that $\psi=\gamma \psi_{0}$, and so $Y_{j}=\gamma Y_{j}^{0}$. Our main objective is the determination of this scalar $\gamma$. Note that the determination of $\gamma$ is equivalent to the determination of the map $\sigma_{\left(C^{\prime}, \mathcal{E}^{\prime}\right)}$. In this paper, we determine $\gamma$ by investigating the map $\sigma_{\left(C^{\prime}, \mathcal{E}^{\prime}\right)}$. The following fact is easily verified.

Lemma 1.11. Suppose that $q^{-\left(a_{0}+r\right) / 2} \sigma_{\left(C^{\prime}, \mathcal{E}^{\prime}\right)}$ makes the $\mathcal{W}$-module $V_{\left(C^{\prime}, \mathcal{E}^{\prime}\right)}$ the preferred extension to $\widetilde{\mathcal{W}}$. Then we have $\gamma=1$. 


\section{$\S 2$. Unipotent classes of classical groups}

2.1. Let $G$ be a connected classical group defined over $\mathbf{F}_{q}$. We consider the following type of groups $G$.

(I) $G=S p_{2 n}, p \neq 2$,

(II) $G=S O_{2 n+1}, p \neq 2$,

(III) $G=S O_{2 n}^{ \pm}, p \neq 2$.

(IV) $G=S p_{2 n}, p=2$,

(V) $G=S O_{2 n}^{ \pm}, p=2$.

These groups are realized as a group of transformations preserving the various forms. Let $V$ be a vector space over $k$ with $\operatorname{dim} V=N$. Assume that $p \neq 2$. Then $S p_{N}$ (resp. $O_{N}$ ) is the subgroup of $G L(V)$ leaving $f$ invariant, where $f$ is an alternating form (resp. a symmetric bilinear form) on $V$ and $N=2 n$ in the case of $S p . S O_{N}$ is the connected component of $O_{N}$, and $S O_{2 n}^{ \pm}$corresponds to two $\mathbf{F}_{q}$-forms of $f$, one is split, the other is non-split.

Assume that $p=2$. Then $S p_{2 n}$ is the subgroup of $G L(V)$ with $N=2 n$ leaving an alternating form (= a symmetric bilinear form) $f$ invariant. The quadratic form $Q$ on $V$ is defined by the property that the map $V \times V \rightarrow$ $k,(x, y) \mapsto Q(x+y)-Q(x)-Q(y)$ gives rise to a non-singular bilinear form, which we may take the alternating form $f$. Let $O_{2 n}$ be the subgroup of $G L(V)$ leaving $Q$ invariant. Then we have $O_{2 n} \subset S p_{2 n}$, and let $S O_{2 n}$ be the connected component of $O_{2 n}$. It is known by [D] that there exists two $\mathbf{F}_{q}$-forms of $Q$ as follows. We regard $Q$ as the quadratic form on $V_{0}=\mathbf{F}_{q}^{2 n}$. Then there exists a basis of $V_{0}$ such that, for $x=\left(x_{1}, \ldots, x_{2 n}\right) \in V_{0}$ with respect to this basis, $Q(x)$ can be expressed as

$$
\begin{aligned}
& Q(x)=x_{1} x_{n+1}+\cdots+x_{n} x_{2 n}, \\
& Q(x)=x_{1} x_{n+1}+\cdots+x_{n-1} x_{2 n-1}+\alpha x_{n}^{2}+x_{n} x_{2 n}+\alpha x_{2 n}^{2},
\end{aligned}
$$

where $\alpha \in \mathbf{F}_{q}$ is an element such that $\alpha X^{2}+X+\alpha$ is an irreducible polynomial in $\mathbf{F}_{q}[X]$. We denote by $O_{2 n}^{+}$(resp. $O_{2 n}^{-}$) the group $O_{2 n}$ associated to the form in (2.1.1) (resp. (2.1.2)), and let $S O_{2 n}^{ \pm}$be the connected component of $O_{2 n}^{ \pm}$.

2.2. We shall describe the unipotent classes in $G$. As is well-known, in the case where $p \neq 2$, the unipotent classes of $G$ are described by unipotent classes in $G L(V)$ which are parametrized by partitions of $N$ through Jordan 
normal form. Let $\widetilde{C}_{\lambda}$ be the unipotent class in $G L(V)$ corresponding to a partition $\lambda$ of $N$. We write $\lambda$ as $\lambda=\left(\lambda_{1} \leq \lambda_{2} \leq \cdots \leq \lambda_{r}\right)$ with $\sum_{i=1}^{r} \lambda_{i}=N$ or $\lambda=\left(1^{c_{1}}, 2^{c_{2}}, \ldots\right)$, where $r=l(\lambda)$ is called the length of $\lambda$. Assume that $G=S p_{2 n}$. Then $C_{\lambda}=\widetilde{C}_{\lambda} \cap G$ is non-empty if and only if $c_{i}$ is even for odd $i$, and in that case $C_{\lambda}$ is a single conjugacy class in $G$. While for $\widetilde{G}=O_{N}$, $C_{\lambda}=\widetilde{C}_{\lambda} \cap \widetilde{G}$ is non-empty if and only if $c_{i}$ is even for even $i$, and in that case $C_{\lambda}$ form a single class in $\widetilde{G}$. Now $C_{\lambda}$ is already contained in $G=S O_{N}$, and so gives a unipotent class in $G$ in almost all cases. The exceptions are the cases where $\lambda$ satisfies the condition; $c_{i}=0$ if $i$ is even, and $c_{i}$ is even for all odd $i$. In that case, $C_{\lambda}$ is divided into two classes $C_{\lambda}^{\prime}$ and $C_{\lambda}^{\prime \prime}$ in $G$.

2.3. In the case where $p=2$, the parametrization of unipotent classes is more complicated. We shall describe it following Spaltenstein [Sp1, 2.6]. First assume that $G=S p_{2 n}$ with $p=2$, and let $f$ be the associated alternating form. Then the unipotent classes in $G$ are parametrized by a pair $(\lambda, \varepsilon)$, where $\lambda$ is a partition of $2 n$ such that $c_{i}$ is even for odd $i$, and $\varepsilon$ is an assignment $\varepsilon: i \mapsto \varepsilon_{i} \in\{0,1\}$ for even $i$ such that $c_{i} \neq 0$. Here $\varepsilon_{i}=1$ if $c_{i}$ is odd, and $\varepsilon_{i}=0$ or 1 if $c_{i}$ is even. The correspondence with unipotent classes are given as follows. Let $u$ be a unipotent element in $G$. Then as an element in $G L(V), u$ is parametrized by a partition $\lambda$ of $2 n$, which satisfies a similar condition as in the case of $p \neq 2$. Now take even $i$ such that $c_{i}$ is even non-zero. We define a function $h_{i}$ on $\operatorname{Ker}(u-1)^{i}$ by $h_{i}(x)=f\left((u-1)^{i-1} x, x\right)$. Then we put

$$
\varepsilon_{i}= \begin{cases}0 & \text { if } h_{i} \equiv 0 \\ 1 & \text { otherwise }\end{cases}
$$

The pair $(\lambda, \varepsilon)$ is the one corresponding to the unipotent class in $G$ containing $u$. We denote by $C_{\lambda, \varepsilon}$ the unipotent class in $G$ corresponding to $(\lambda, \varepsilon)$. For a convenience sake, we extend $\varepsilon$ to the function on $\mathbf{N}$ by $\varepsilon: i \mapsto \varepsilon_{i}$, where $\varepsilon_{i}=\omega$ for $i$ not appeared above ( $\omega$ is a symbol not contained in $\{0,1\})$.

Next assume that $G=S O_{2 n}$ with $p=2$. We have $\widetilde{G}=O_{2 n} \subset S p_{2 n}$. Let $\widetilde{C}_{\lambda, \varepsilon}$ be the unipotent class in $S p_{2 n}$ corresponding to $(\lambda, \varepsilon)$. Then $C_{\lambda, \varepsilon}=$ $\widetilde{C}_{\lambda, \varepsilon} \cap \widetilde{G}$ is a unipotent class in $\widetilde{G}$. Thus unipotent classes in $\widetilde{G}$ are in 1:1 correspondence with unipotent classes in $S p_{2 n}$. Now $C_{\lambda, \varepsilon}$ is contained in $G$ if and only if $l(\lambda)$ is even. Assume that $l(\lambda)$ is even. Then $C_{\lambda, \varepsilon}$ forms a single unipotent class in $G$ except for the case where $c_{i}=0$ for all odd $i$, and $\varepsilon_{i}=0$ for all even $i$ such that $c_{i} \neq 0$ (here $c_{i}$ is even for even $i$ ). In the latter case, $C_{\lambda, \varepsilon}$ splits into two classes $C_{\lambda, \varepsilon}^{\prime}$ and $C_{\lambda, \varepsilon}^{\prime \prime}$ in $G$. 
2.4. Let $G$ be as in 2.1. For a convenience sake, we introduce a function $\varepsilon$ on $\mathbf{N}$ also in the case of $p \neq 2$. Assume that $p \neq 2$. In the case of $G=S p_{2 n}$, we put $\varepsilon_{i}=1$ if $i$ is even and $c_{i} \neq 0$, and put $\varepsilon_{i}=\omega$ otherwise. In the case of $O_{N}$, we put $\varepsilon_{i}=1$ if $i$ is odd and $c_{i} \neq 0$, and put $\varepsilon_{i}=\omega$ otherwise. Under this convention, we denote the class $C_{\lambda}$ in $S p_{2 n}$ or $S O_{N}$ by $C_{\lambda, \varepsilon}$. For $u \in G$, let $A_{G}(u)$ be the component group of $Z_{G}(u)$ as before. In the case of $\widetilde{G}=O_{N}$, we also consider $A_{\widetilde{G}}(u)=Z_{\widetilde{G}}(u) / Z_{\widetilde{G}}^{0}(u)$ for $u \in \widetilde{G}$. Following [Sp1, 2.9], we shall describe the structure of $A_{G}(u)$ and $A_{\widetilde{G}}(u)$.

Assume that $G=S p_{2 n}$ with $p \neq 2$. Take $u \in C_{\lambda, \varepsilon}$. We consider the generator $a_{i}$ corresponding to each $\lambda_{i}$. Then $A_{G}(u)$ is an abelian group generated by $a_{i}$ such that $\varepsilon\left(\lambda_{i}\right)=1$ under the condition that $a_{i}^{2}=1$ and that $a_{i}=a_{j}$ if $\lambda_{i}=\lambda_{j}$.

Next assume that $\widetilde{G}=O_{N}$ with $p \neq 2$. Take $u \in C_{\lambda, \varepsilon}$. Then $A_{\widetilde{G}}(u)$ is an abelian group generated by $a_{i}$, exactly by the same condition as the case of $S p_{2 n}$. Now $A_{G}(u)$ is the subgroup of $A_{\widetilde{G}}(u)$ of index 2 generated by $a_{i} a_{j}$ for each $i \neq j$.

Next assume that $G=S p_{2 n}$ with $p=2$. Take $u \in C_{\lambda, \varepsilon}$. Again we consider the generators $a_{i}$ corresponding to $\lambda_{i}$. Then $A_{G}(u)$ is an abelian group generated by $a_{i}$ such that $\varepsilon\left(\lambda_{i}\right) \neq 0$ under the condition that $a_{i}^{2}=1$ and that $a_{i}=a_{j}$ if $\lambda_{i}=\lambda_{j}$ or if $\lambda_{i}=\lambda_{j}+1$ or if $\lambda_{i}$ is even and $\lambda_{i}=\lambda_{j}+2$.

Finally assume that $\widetilde{G}=O_{2 n}$ with $p=2$, and $G=S O_{2 n}$. Take $u \in C_{\lambda, \varepsilon}$. Then $A_{\widetilde{G}}(u)$ is an abelian group generated by $a_{i}$, exactly by the same condition as the case of $S p_{2 n}$ with $p=2$. $A_{G}(u)$ is the subgroup of $A_{\widetilde{G}}(u)$ of index 2 generated by $a_{i} a_{j}$ for $i \neq j$.

2.5. In what follows, we shall construct a normal form of unipotent elements in $G^{F}$. As a preliminary for this, we consider the case where $G=S O_{2 n}$ with $p=2$. So assume given a vector space $V$ over $\mathbf{F}_{q}$ of dimension $N=2 n$ with a basis $e_{1}, \ldots, e_{N}$, endowed with an alternating form $f$. We define an element $v \in G L(V)$ by $(v-1) e_{j}=e_{j-1}$ (with a convention $e_{0}=0$ ), and assume that $v$ leaves $f$ invariant. We consider the following condition on $f$.

$$
\begin{aligned}
& f\left(e_{1}, e_{N}\right)=1, \\
& f\left(e_{i}, e_{N}\right)=0 \text { for } i=n+1, \ldots, N, \\
& f\left(e_{i}, e_{k}\right)+f\left(e_{i+1}, e_{k}\right)+f\left(e_{i}, e_{k+1}\right)=0 \quad \text { for } 0 \leq i, k \leq N-1 .
\end{aligned}
$$

Note that (2.5.3) is equivalent to the condition that $v$ leaves $f$ invariant. Also note that the conditions (2.5.1)-(2.5.3) determines the alternating form 
$f$ invariant by $v$ uniquely. In fact, it follows from (2.5.2) and (2.5.3) that

$$
f\left(e_{i}, e_{j}\right)=0 \text { for } n+1 \leq i, j \leq N .
$$

Also it follows from (2.5.1) and (2.5.3), we have

$$
f\left(e_{i}, e_{j}\right)= \begin{cases}0 & \text { if } i+j \leq N \\ 1 & \text { if } i+j=N+1\end{cases}
$$

Hence it is enough to show that $f\left(e_{i}, e_{j}\right)$ is determined for $1 \leq i \leq n$ and $n+1 \leq j \leq N$. By $(2.5 .5)$ we have $f\left(e_{n}, e_{n+1}\right)=1$. Since $f\left(e_{n+1}, e_{j}\right)=0$ for $j \geq n+1$, we have $f\left(e_{n}, e_{j}\right)=1$ for $j \geq n+1$ by (2.5.3). Then $f\left(e_{i}, e_{j}\right)=$ $f\left(e_{i}, e_{j-1}\right)+f\left(e_{i+1}, e_{j-1}\right)$ is determined for $1 \leq i \leq n$ by induction on $j$ $(n+1 \leq j \leq N)$.

We consider a quadratic form $Q$ such that $Q(x+y)-Q(x)-Q(y)=$ $f(x, y)$, which is left invariant by $v$. We have the following lemma.

Lemma 2.6. Let the notations be as above. Assume that $Q\left(e_{N}\right)=0$. Then $Q$ is determined uniquely, which is non-degenerate of split type.

Proof. Since $Q$ is invariant by $v$, it is known by [Sp1, 6.10] that $Q\left(e_{i}\right)=$ $f\left(e_{i}, e_{i+1}\right)$ for $i=1, \ldots, N-1$. Hence $Q$ is determined uniquely by $f$ and by the condition $Q\left(e_{N}\right)=0$. It is easy to see that this $Q$ actually gives rise to a quadratic form invariant by $v$. In order to show that $Q$ is non-degenerate of split type, it is enough to see that there exists a basis $e_{1}^{\prime}, \ldots, e_{N}^{\prime}$ of $V$ satisfying the property

$$
\begin{gathered}
Q\left(e_{i}^{\prime}\right)=0 \quad \text { for } i=1, \ldots, N, \\
f\left(e_{i}^{\prime}, e_{j}^{\prime}\right)= \begin{cases}1 & \text { if } i+j=N+1, \\
0 & \text { otherwise. }\end{cases}
\end{gathered}
$$

We show (2.6.1). We consider the square matrix $A=\left(f\left(e_{i}, e_{N-j+1}\right)\right)_{1 \leq i, j \leq n}$ of degree $n$. By (2.5.5) $A$ is a lower unitriangular matrix. For $k=0,1, \ldots$, we denote by $A_{k}$ the principal minor matrix of $A$ of degree $2^{k}$. We have $A_{0}=(1)$. We show that

(2.6.2) The matrix $A_{k}$ has the following property; for $k$ such that $2^{k+1} \leq n$, we have

$$
A_{k+1}=\left(\begin{array}{cc}
A_{k} & 0 \\
A_{k} & A_{k}
\end{array}\right) \text {. }
$$


If $2^{k}<n<2^{k+1}, A$ is of the form

$$
A=\left(\begin{array}{cc}
A_{k} & 0 \\
A_{k}^{\prime} & A_{k}^{\prime \prime}
\end{array}\right),
$$

where $A_{k}^{\prime}$ is the minor matrix of $A_{k}$ of type $\left(n-2^{k}, 2^{k}\right)$ consisting of the first $\left(n-2^{k}\right)$-rows and all the columns, and $A_{k}^{\prime \prime}$ is the principal minor matrix of $A_{k}$ of degree $n-2^{k}$.

In fact, assume that $2^{k+1} \leq n$. By induction we may assume that (2.6.2) holds for $k-1$. Put $A=\left(a_{i j}\right)$ with $a_{i j}=f\left(e_{i}, e_{N-j+1}\right)$. Then by (2.5.3), we have

$$
a_{i, j}=a_{i-1, j}+a_{i-1, j-1} .
$$

By induction, we see that the last row of $A_{k}$ is of the form $(1, \ldots, 1)$. Hence (2.6.3) implies that the $\left(2^{k}+1\right)$-th row of $A_{k+1}$ is of the form $(1,0, \ldots, 0,1,0, \ldots, 0)$ (1 appears in the first and the $\left(2^{k}+1\right)$-th coordinates), which coincides with the first row of the matrix $\left(A_{k}, A_{k}\right)$. Since the $\left(2^{k}+2\right)$-th row of $A_{k+1}$ is determined by $\left(2^{k}+1\right)$-th row by (2.6.3), and so on, we see that the minor matrix of $A_{k+1}$ of type $\left(2^{k}, 2^{k+1}\right)$, consisting of the last $2^{k}$-rows and all the columns, coincides with $\left(A_{k}, A_{k}\right)$. (Note that since the last column of $A_{k}$ is of the form ${ }^{t}(0, \ldots, 0,1)$, the interaction between two $A_{k}$ does not occur in this computation). Thus (2.6.2) holds for the case where $2^{k+1} \leq n$. The case where $2^{k}<n<2^{k+1}$ is dealt similarly.

For $j$ such that $2^{a-1}<j \leq 2^{a}$, we define a marked matrix $A^{(j)}$ as follows. In the matrix $A_{a}$, the $(j, j)$ entry is contained in a minor matrix $A_{1}=\left(\begin{array}{ll}1 & 0 \\ 1 & 1\end{array}\right)$, where the $(j, j)$-entry corresponds to the $(1,1)$-entry (resp. $(2,2)$-entry) of $A_{1}$ if $j$ is odd (resp. even). We define a marked matrix $A_{a}^{(j)}$ by replacing the minor matrix $A_{1}$ in $A_{a}$ by $A_{1}^{\bullet}$, where

$$
A_{1}^{\bullet}=\left(\begin{array}{cc}
1^{\bullet} & 0 \\
1^{\bullet} & 1^{\bullet}
\end{array}\right) \quad \text { or } \quad A_{1}^{\bullet}=\left(\begin{array}{cc}
1 & 0 \\
1 & 1
\end{array}\right)
$$

according as $(j, j)$ corresponds to $(1,1)$ or $(2,2)$ in $A_{1}$. In each of the matrices the marks $\bullet$ are attached to some entries in $A_{a}$. For example, for $2<j \leq 2^{2}, A_{2}^{(j)}$ is given as

$$
\left(\begin{array}{cccc}
1 & 0 & 0 & 0 \\
1 & 1 & 0 & 0 \\
1 & 0 & 1^{\bullet} & 0 \\
1 & 1 & 1^{\bullet} & 1^{\bullet}
\end{array}\right) \quad \text { or } \quad\left(\begin{array}{cccc}
1 & 0 & 0 & 0 \\
1 & 1 & 0 & 0 \\
1 & 0 & 1 & 0 \\
1 & 1 & 1 & 1^{\bullet}
\end{array}\right)
$$


according to the case where $j=3$ or $j=4$. For $k>a$, we define $A_{k}^{(j)}$ inductively as in (2.6.2) by replacing $A_{k}$ by $A_{k}^{(j)}$, starting from $A_{a}^{(j)}$. Then we define the matrix $A^{(j)}$ for $2^{k} \leq n<2^{k+1}$ by replacing $A_{k}^{\prime}$, $A_{k}^{\prime \prime}$ by $\left(A_{k}^{(j)}\right)^{\prime}$, $\left(A_{k}^{(j)}\right)^{\prime \prime}$ which is defined similarly.

By a direct observation, we have

(2.6.4) The matrix $A^{(j)}$ has the following properties.

(i) In each row, the number of marked 1 is even except the $j$-th row, where the number is 1 .

(ii) In each column containing the marked 1's, the entries except the marked 1 are all zero.

We now define, for $j=1, \ldots, n$, the vector $e_{N-j+1}^{\prime}$ by

$$
e_{N-j+1}^{\prime}=\sum_{k} e_{N-k+1}
$$

where the sum is taken over $1 \leq k \leq n$ such that $k$-th column in $A^{(j)}$ contains a marked 1. It follows from (2.6.4) that we have

$$
f\left(e_{i}, e_{N-j+1}^{\prime}\right)= \begin{cases}1 & \text { if } i=j, \\ 0 & \text { otherwise }\end{cases}
$$

for $1 \leq i, j \leq n$.

We now consider the values of $Q$. Since $Q$ satisfies the relation $Q\left(e_{i}\right)=$ $f\left(e_{i}, e_{i+1}\right)$, it follows, by (2.5.4) and (2.5.5) together with our assumption that $Q\left(e_{N}\right)=0$, that

$$
Q\left(e_{i}\right)= \begin{cases}0 & \text { if } i \neq n, \\ 1 & \text { if } i=n .\end{cases}
$$

Note that we have $e_{n+1}^{\prime}=e_{n+1}$ by the previous computation. Put $e_{n}^{\prime}=$ $e_{n}+e_{n+1}$. Then we have

$$
\begin{aligned}
Q\left(e_{n}^{\prime}\right) & =Q\left(e_{n}\right)+Q\left(e_{n+1}\right)+f\left(e_{n}, e_{n+1}\right)=0, \\
f\left(e_{n}^{\prime}, e_{n+1}^{\prime}\right) & =f\left(e_{n}+e_{n+1}, e_{n}\right)=1 .
\end{aligned}
$$

Now put $e_{i}^{\prime}=e_{i}$ for $i=1, \ldots, n-1$. Then by (2.5.4) and (2.5.5), together with (2.6.5)-(2.6.7), we see that the basis $\left\{e_{1}^{\prime}, \ldots, e_{N}^{\prime}\right\}$ satisfies the relation (2.6.1). The lemma is proved. 
2.7. Let $G$ be as in 2.1. We assume that $G^{F}$ is of split type. For each $F$-stable unipotent class $C$ in $G$, we shall construct a normal form $u$, called a split element, in $C^{F}$. The $G^{F}$-conjugacy class of $u$ is called the split class in $C^{F}$. In the case where $p=2$, we construct $u$ following [Sp1, II, 6.19]. First consider the case where $G=S p_{2 n}$ with $p=2$. Take a unipotent class $C_{\lambda, \varepsilon}$ of $G$. For each $j \geq 1$, put

$$
n_{j}= \begin{cases}\lambda_{j} & \text { if } \varepsilon\left(\lambda_{j}\right)=1, \\ \lambda_{j}+1 & \text { if } \varepsilon\left(\lambda_{j}\right)=\varepsilon, \\ \lambda_{j}+2 & \text { if } \varepsilon\left(\lambda_{j}\right)=0 .\end{cases}
$$

We consider the vector space $V_{j}$ over $\mathbf{F}_{q}$ of dimension $n_{j}$ with basis $e_{1}^{j}, \ldots$, $e_{n_{j}}^{j}$. Assume that a non-degenerate alternating form (= a symmetric bilinear form) $f_{j}$ on $V_{j}$ is given. Let $H\left(V_{j}\right)$ be the subgroup of $G L\left(V_{j}\right)$ consisting of $g \in G L\left(V_{j}\right)$ which leaves the form $f_{j}$ invariant. Put $H=S p_{n_{j}}(k)$. Then $H\left(V_{j}\right)$ is regarded as a subgroup $H^{F}$ of $H$ under the natural $\mathbf{F}_{q^{-}}$-structure $F$ on $H$.

One can construct $v_{j} \in H\left(V_{j}\right)$ such that $\left(v_{j}-1\right) e_{i}^{j}=e_{i-1}^{j}$ for $i=$ $1, \ldots, n_{j}$ (under the convention that $e_{0}^{j}=0$ ) with respect to the alternating form $f_{j}$ satisfying the property as given in 2.5 (with $f=f_{j}, N=n_{j}$ ).

We also note that

(2.7.2) The image of $v_{j} \in Z_{H}\left(v_{j}\right)$ to $A_{H}\left(v_{j}\right)$ gives a generator $\bar{a}_{j}$ of $A_{H}\left(v_{j}\right)$, where $A_{H}\left(v_{j}\right)$ is of order 1 or 2 .

For each $h=\lambda_{j}$, we shall construct a vector space $M_{j}$ over $\mathbf{F}_{q}$ with an alternating form $f_{j}^{0}$, and $u_{j} \in H\left(M_{j}\right)$ as follows.

(a) $\varepsilon(h)=1$. In this case, $h$ is even and $c_{h}$ is odd or even. We put $M_{j}=V_{j}$ and $u_{j}=v_{j}$. Thus $u_{j} \in H\left(M_{j}\right)$ with respect to $f_{j}^{0}=f_{j}$ on $M_{j}$. Since $f_{j}^{0}\left(e_{n_{j}}^{j}, e_{1}^{j}\right)=1$, we see that the function $x \mapsto f_{j}^{0}\left(\left(u_{j}-1\right)^{h-1} x, x\right)$ is non-trivial on $\operatorname{Ker}\left(u_{j}-1\right)^{h}$.

(b) $\varepsilon(h)=\omega$. In this case $h$ is odd and $c_{h}$ is even. Assume that $\lambda_{j}=\lambda_{j-1}=h$, and let $\left(V_{j}, f_{j}\right)$ and $\left(V_{j-1}, f_{j-1}\right)$ be as before. Note that $\operatorname{dim} V_{j}=\operatorname{dim} V_{j-1}=n_{j}=h+1$ by (2.7.1). We define an alternating form $f^{\prime}$ on $V_{j} \oplus V_{j-1}$ by the condition that $\left.f^{\prime}\right|_{V_{j}}=f_{j},\left.f^{\prime}\right|_{V_{j-1}}=f_{j-1}$ and that $V_{j} \perp V_{j-1}$. Let $L$ be a line in $V_{j} \oplus V_{j-1}$ generated by $e_{1}^{j}+e_{1}^{j-1}$. Then $L$ is an isotropic line by (2.5.5) and we put $M_{j}=L^{\perp} / L$. We have $\operatorname{dim} M_{j}=2 h$. 
Since $v_{j}\left(e_{1}^{j}\right)=e_{1}^{j}, v_{j-1}\left(e_{1}^{j-1}\right)=e_{1}^{j-1}$, we see that $v_{j}+v_{j-1}$ fixes $L$, and so it induces a linear transformation on $M_{j}$, which we denote by $u_{j}$. The form $f^{\prime}$ induces an alternating form $f_{j}^{0}$. We have $u_{j} \in H\left(M_{j}\right)$.

By (2.5.1) and (2.5.5), $L^{\perp}$ has a basis

$$
e_{n}^{j}+e_{n}^{j-1}, e_{n-1}^{j}, e_{n-1}^{j-1}, \ldots, e_{2}^{j}, e_{2}^{j-1}, e_{1}^{j}+e_{1}^{j-1} .
$$

Hence $L^{\perp} / L$ has a basis

$$
\bar{e}_{n}^{j}+\bar{e}_{n}^{j-1}, \bar{e}_{n-1}^{j}, \bar{e}_{n-1}^{j-1}, \ldots, \bar{e}_{2}^{j}, \bar{e}_{2}^{j-1}, \bar{e}_{1}^{j}=\bar{e}_{1}^{j-1}
$$

where $\bar{e}_{i}^{j}, \bar{e}_{i}^{j-1}$ denote the image of $e_{i}^{j}, e_{i}^{j-1}$ on $\left(V_{j} \oplus V_{j-1}\right) / L$.

(c) $\varepsilon(h)=0$. In this case, $h$ is even and $c_{h}$ is even. Assume that $\lambda_{j}=\lambda_{j-1}$, and consider the vector spaces $V_{j}$ and $V_{j-1}$ as before. By (2.7.1), we have $n_{j}=\operatorname{dim} V_{j}=\operatorname{dim} V_{j-1}=h+2$. We consider the alternating form $f^{\prime}$ on $V_{j} \oplus V_{j-1}$ as before. Let $N$ be the subspace of $V_{j} \oplus V_{j-1}$ spanned by $e_{1}^{j}+e_{1}^{j-1}$ and $e_{2}^{j}+e_{2}^{j-1}$. Then $N$ is an isotropic subspace of $V_{j} \oplus V_{j-1}$ of dimension 2, and we put $M_{j}=N^{\perp} / N$. We have $\operatorname{dim} M_{j}=2 h$. The alternating form $f^{\prime}$ induces an alternating form $f_{j}^{0}$ on $M_{j}$. Now $v_{j}+v_{j-1}$ stabilized $N$, and so induces a linear transformation on $M_{j}$ which we denote by $u_{j}$. We see that $u_{j} \in H\left(M_{j}\right)$.

By (2.5.1) and (2.5.5), $N^{\perp}$ has a basis

$$
e_{n}^{j}+e_{n}^{j-1}, e_{n-1}^{j}+e_{n-1}^{j-1}, e_{n-2}^{j}, e_{n-2}^{j-1}, \ldots, e_{1}^{j}, e_{1}^{j-1} .
$$

Hence $N^{\perp} / N$ has a basis

$$
\bar{e}_{n}^{j}+\bar{e}_{n}^{j-1}, \bar{e}_{n-1}^{j}+\bar{e}_{n-1}^{j-1}, \bar{e}_{n-2}^{j}, \bar{e}_{n-2}^{j-1}, \ldots, \bar{e}_{3}^{j}, \bar{e}_{3}^{j-1}, \bar{e}_{2}^{j}=\bar{e}_{2}^{j-1}, \bar{e}_{1}^{j}=\bar{e}_{1}^{j-1},
$$

where $\bar{e}_{i}^{j}, \bar{e}_{i}^{j-1}$ denotes the image of $e_{i}^{j}$ on $\left(V_{j} \oplus V_{j-1}\right) / N$. The action of $u_{j}$ on this basis is easily described, and by using the formulas in 2.5, one can check that $f_{j}^{0}\left(\left(u_{j}-1\right)^{h-1} x, x\right)=0$ for all $x \in \operatorname{Ker}\left(u_{j}-1\right)^{h}$. For example,

$$
f_{j}^{0}\left(\bar{e}_{n}^{j}+\bar{e}_{n}^{j-1}, \bar{e}_{3}^{j}+\bar{e}_{3}^{j-1}\right)=2 f_{j}\left(e_{n}^{j}, e_{3}^{j}\right)=0
$$

and the other cases are dealt similarly.

We now define a vector space $\bar{V}$ as $\bar{V}=\bigoplus_{j} M_{j}$ so that $\operatorname{dim} \bar{V}=2 n$, and let $f=\bigoplus_{j} f_{j}^{0}$ be the alternating form obtained from $f_{j}^{0}$. Put $\bar{u}=\prod_{j} u_{j} \in$ $H(\bar{V})$. It follows from the previous construction, we have 
(2.7.3) $H(\bar{V})$ can be identified with $G^{F}$, and under this isomorphism, the element $u \in G^{F}$ corresponding to $\bar{u}$ gives an element in $C_{\lambda, \varepsilon}^{F}$. We call $u$ a split element in $C_{\lambda, \mathcal{E}}^{F}$.

The structure of the group $A_{G}(u)$ is also described as follows (cf. [Sp1, II, 6.19]) Take $\lambda_{j}$ such that $\varepsilon\left(\lambda_{j}\right) \neq 0$. We denote by $\bar{a}_{j}$ an automorphism of $\bigoplus_{k>1} V_{k}$ defined by $v \mapsto v_{j}(v)$ for $v \in V_{j}$, and $v \mapsto v$ for $v \in V_{k}$ such that $k \neq j$. Then $\bar{a}_{j}$ induces an automorphism on $\bar{V}$ commuting with $\bar{u}$, which we denote also by $\bar{a}_{j}$. It is checked that $\bar{a}_{j} \in H(\bar{V})$, and so this gives an element of $Z_{G}(u)$. The image of $\bar{a}_{j}$ on $A_{G}(u)$ coincides with the generator $a_{j}$ stated in 1.4. In particular, we see that $F$ acts trivially on $A_{G}(u)$. This implies, since $A_{G}(u)$ is abelian, that

(2.7.4) For any $u^{\prime} \in C_{\lambda, \varepsilon}^{F}, F$ acts trivially on $A_{G}\left(u^{\prime}\right)$.

2.8. Next we consider the case where $G=S O_{2 n}$ with $p=2$. Take a unipotent class $C_{\lambda, \varepsilon}$ of $G$, and let $n_{j}$ be as in (2.7.1). We consider the vector space $V_{j}$ over $\mathbf{F}_{q}$ with basis $e_{1}^{j}, \ldots, e_{n_{j}}^{j}$ and a unipotent element $v_{j} \in G L\left(V_{j}\right)$ as in 2.7. By Lemma 2.6, one can construct a split quadratic form $Q_{j}$ on $V_{j}$ which is invariant by $v_{j}$. Let $H\left(V_{j}\right)$ be the subgroup of $G L\left(V_{j}\right)$ consisting of $g$ which leaves $Q_{j}$ invariant. Let $\widetilde{H}=O_{n_{j}}(k)$. Since $Q_{j}$ is of split type, $H\left(V_{j}\right)$ can be identified with the subgroup $\widetilde{H}^{F}$ of $\widetilde{H}$, where $F$ is a split Frobenius map. Then by a similar argument as in 2.7, we obtain $u_{j} \in H\left(M_{j}\right)$ for each case (a), (b) or (c), where $H\left(M_{j}\right)$ is the group of invariants with respect to the induced quadratic from $Q_{j}^{0}$. Note that the explicit computation in the proof of Lemma 2.6 shows that $Q_{j}^{0}$ is of split type. As in 2.7, we define a vector space $\bar{V}=\bigoplus_{j} M_{j}$ and $Q=\bigoplus_{j} Q_{j}^{0}$, and put $\bar{u}=\prod_{j} u_{j} \in H(\bar{V})$. We have

(2.8.1) Let $\widetilde{G}=O_{2 n}^{+}$. Then $H(\bar{V})$ can be identified with $\widetilde{G}^{F}$ with split Frobenius map $F$, and under this isomorphism, the element $u \in \widetilde{G}^{F}$ corresponding to $\bar{u}$ gives an element in $\widetilde{C}_{\lambda, \varepsilon}^{F}$. In the ordinary case $u \in C_{\lambda, \varepsilon}^{F}$. In the exceptional case we have $u \in\left(C_{\lambda, \varepsilon}^{\prime}\right)^{F}$ and $\sigma(u) \in\left(C_{\lambda, \varepsilon}^{\prime \prime}\right)^{F}$, where $\sigma$ is the graph automorphism on $S_{2 n}$. We call $u$ and $\sigma(u)$ the split elements in $\widetilde{C}_{\lambda}^{F}$.

2.9. Next we consider the case where $G=S p_{2 n}$ or $S O_{N}$ with $p \neq 2$. We assume that $F$ is a split Frobenius map. Let $\mathfrak{g}$ be the Lie algebra of $G$. Since the unipotent classes in $G$ are in bijection with the nilpotent orbits in $\mathfrak{g}$ with $\mathbf{F}_{q}$-structure, we consider the normal form of nilpotent orbits instead 
of unipotent classes. Let $\mathcal{O}_{\lambda, \varepsilon}$ be the nilpotent orbit in $\mathfrak{g}$ corresponding to the unipotent class $C_{\lambda, \varepsilon}$ in $G$. For each $\lambda_{j}$, we construct a vector space $M_{j}$ over $\mathbf{F}_{q}$ and a nilpotent transformation $X_{j}$ on $M_{j}$ as follows.

(a) $\varepsilon\left(\lambda_{j}\right)=1$. We consider a vector space $M_{j}$ of dimension $h=\lambda_{j}$ with basis $e_{1}^{j}, \ldots, e_{h}^{j}$. We define a non-degenerate alternating form (resp. a symmetric bilinear form) $f_{j}$ on $M_{j}$ in the case where $G=S p_{2 n}\left(\right.$ resp. $\left.S O_{N}\right)$ by

$$
f_{j}\left(e_{h-i+1}^{j}, e_{i}^{j}\right)=(-1)^{\delta_{j}-i} \quad \text { for } i=1, \ldots, h,
$$

where

$$
\delta_{j}= \begin{cases}\lambda_{j} / 2+j & \text { if } G=S p_{2 n}, \\ \left(\lambda_{j}-1\right) / 2+j & \text { if } G=S O_{N} .\end{cases}
$$

We put the value of $f_{j}$ zero for any other pair of the basis. We define a nilpotent transformation $X_{j}$ on $M_{j}$ by $X_{j}\left(e_{i}^{j}\right)=e_{i-1}^{j}$ (under the convention that $\left.e_{0}=0\right)$. Then $X_{j} \in \mathfrak{h}\left(M_{j}\right)$, where $\mathfrak{h}\left(M_{j}\right)$ is the subalgebra of $\mathfrak{g l}\left(M_{j}\right)$ consisting of $X$ such that $f_{j}(X x, y)+f_{j}(x, X y)=0$ for $x, y \in M_{j}$.

(b) $\varepsilon\left(\lambda_{j}\right)=\omega$. In this case $c_{h}$ is even for $\lambda_{j}=h$. We assume that $\lambda_{j}=\lambda_{j-1}$. We consider a vector space $M_{j}$ of dimension $2 h=2 \lambda_{j}$ with basis $e_{1}^{j}, \ldots, e_{h}^{j}, e_{1}^{j-1}, \ldots, e_{h}^{j-1}$. We define an alternating form (resp. a symmetric bilinear form) $f_{j}$ on $M_{j}$ in the case where $G=S p_{2 n}\left(\right.$ resp. $\left.G=S O_{N}\right)$ by

$$
f_{j}\left(e_{h-i+1}^{j}, e_{i}^{j-1}\right)=\varepsilon f_{j}\left(e_{i}^{j-1}, e_{h-i+1}^{j}\right)=(-1)^{i-1} \quad \text { for } i=1, \ldots, h,
$$

where $\varepsilon=-1$ (resp. $\varepsilon=1)$ if $G=S p_{2 n}$ (resp. $\left.G=S O_{N}\right)$. We put the values of $f_{j}$ zero for any other pair of the basis. We define a nilpotent transformation $X_{j}$ on $M_{j}$ by $X_{j} e_{i}^{j}=e_{i-1}^{j}, X_{j} e_{i}^{j-1}=e_{i-1}^{j-1}$ (we put $e_{0}^{j}=$ $e_{0}^{j-1}=0$ as before). Then $X_{j} \in \mathfrak{h}\left(M_{j}\right)$.

We define a vector space $\bar{V}$ by $\bar{V}=\bigoplus_{j} M_{j}$ so that $\operatorname{dim} \bar{V}=N$, and let $f=\sum_{j} f_{j}$ be an alternating form (resp. a symmetric bilinear form) on $\bar{V}$ obtained from $f_{j}$. Put $\bar{X}=\bigoplus_{j} X_{j} \in \mathfrak{h}(\bar{V})$. Then it is known by [SS],

(2.9.3) $\mathfrak{h}(\bar{V})$ can be identified with $\mathfrak{g}^{F}$. Under this correspondence $\bar{X}$ gives an element $X \in \mathcal{O}_{\lambda, \varepsilon}^{F}$, which we call a split element in $\mathcal{O}_{\lambda, \varepsilon}^{F} . X$ also determines the $G^{F}$-class in $C_{\lambda, \varepsilon}^{F}$, which we call the split class in $C_{\lambda, \varepsilon}^{F}$. 
2.10. We consider the case where $G=S O_{2 n}$ with non-split Frobenius $F$ (for arbitrary $p$ ). Let $F_{0}$ be the split Frobenius map. Then one can write $F=F_{0} \sigma$ with the graph automorphism $\sigma$. We may choose $s \in \widetilde{G} \backslash G$ such that $\sigma=\operatorname{ad} s$, and fix it for all. Let $u \in G^{F_{0}}$ be a split element in $C_{\lambda, \varepsilon}$. Since $A_{\widetilde{G}}(u) \neq A_{G}(u)$, there exists $a \in A_{\widetilde{G}}(u) \backslash A_{G}(u)$. Let $\dot{a} \in Z_{\widetilde{G}}(u)$ be a representative of $a$. Since $[\widetilde{G}: G]=2$, there exists $g \in G$ such that $\dot{a}=g s$. It follows that ${ }^{g F} u=u$. Now there exists $\alpha \in G$ such that $\alpha^{-1} F(\alpha)=g$, and we have $u^{\prime}=\alpha u \alpha^{-1} \in C_{\lambda, \varepsilon}^{F}$. It is easy to see that the $G^{F}$-conjugacy class of $u^{\prime}$ is uniquely determined by $\dot{a} \in Z_{\widetilde{G}}(u)$. For the exceptional case, we have $u^{\prime} \in\left(C_{\lambda, \varepsilon}^{\prime}\right)^{F}$ and $\sigma\left(u^{\prime}\right) \in\left(C_{\lambda, \varepsilon}^{\prime \prime}\right)^{F}$. In what follows, we fix a split element $u^{\prime} \in C_{\lambda, \varepsilon}^{F}$ as follows.

(2.10.1) Let $G=S O_{2 n}^{-}$. Let $u$ be the split element in $C_{\lambda, \varepsilon}^{F_{0}}$ or $\left(C_{\lambda, \varepsilon}^{\prime}\right)^{F_{0}}$. We choose $a=a_{i} \in A_{\widetilde{G}}(u)$ such that $\varepsilon\left(\lambda_{i}\right)=1$ (resp. $\varepsilon\left(\lambda_{i}\right) \neq 0$ ) in the case where $p \neq 2$ (resp. $p=2$ ) and that $\lambda_{i}$ is minimal under this condition. Take a representative $\dot{a}_{i} \in Z_{\widetilde{G}}(u)$ as in 2.7 , and define $u^{\prime} \in C_{\lambda, \varepsilon}^{F}$ by using $\dot{a}_{i}$. (In the exceptional case, define $u^{\prime} \in\left(C_{\lambda, \varepsilon}^{\prime}\right)^{F}$ and $\sigma\left(u^{\prime}\right) \in\left(C_{\lambda, \varepsilon}^{\prime \prime}\right)^{F}$.) We call $u^{\prime}$ the split element in $C_{\lambda, \varepsilon}^{F}$.

Under the notation above, $a F_{0}=g F$ acts trivially on $A_{G}(u)$ by (2.7.4). It follows that $F$ acts trivially on $A_{G}\left(u^{\prime}\right)$. Since $A_{G}\left(u^{\prime}\right)$ is abelian, we have (2.10.2) The statement (2.7.4) holds also for the case where $F$ is of non-split type.

Remark 2.11. The definition of the split elements for $S p_{2 n}$ or $S O_{N}$ (for $p \neq 2$ ) in $2.9,2.10$ is essentially the same as the one used in [S1, 3.3, 3.7] (where they are called distinguished elements). Note that in the case where $q \equiv 1(\bmod 4), \delta_{j}$ can be removed in the formula $(2.9 .1)$ by a suitable base change. Also note that the definition of $f_{j}$ involves the case where $\varepsilon(h)=1$ and $c_{h}$ is even, which is necessary for later discussions, though these cases are ignored in [S1].

In the case of non-split groups with $p \neq 2$, our definition of split elements is not the same as in [S1, 3.7], where it is defined by using $a=a_{i}$ corresponding to $\lambda_{i}$ of maximal length instead of minimal length. This is not essential, but the definition here is more convenient since it produces preferred extensions of $\mathcal{W}$-modules as will be seen in Theorem 4.3. (The elements defined in [S1, 3.7] do not necessarily produce them). 


\section{$\S 3$. Generalized Springer correspondence}

3.1. We review here the generalized Springer correspondence for classical groups following [L1] and [LS]. In what follows, we denote by $W_{n}$ the Weyl group of type $C_{n}$, and by $W_{n}^{\prime}$ the Weyl group of type $D_{n}$. Throughout the whole cases, for a given $(L, C, \mathcal{E}) \in \mathcal{M}_{G}$, the cuspidal pair $(C, \mathcal{E})$ is uniquely determined by $L$. So, we just describe $L$ which has a cuspidal pair.

(a) Let $G=S p_{2 n}$ with $p \neq 2$. Then $(L, C, \mathcal{E}) \in \mathcal{M}_{G}$ if and only if $L$ is of type $C_{m}$ for some $m$ of the form $m=\frac{1}{2} d(d-1)$ with $d \geq 1$. We have $N_{G}(L) / L \simeq W_{n-\frac{1}{2} d(d-1)}$. Since the set $\{d(d-1) \mid d \geq 1\}$ coincides with the set $\{d(d-1) \mid d \in \mathbf{Z}, d$ : odd $\}$, the generalized Springer correspondence (1.1.3) is given by a bijection

$$
\mathcal{N}_{G} \longleftrightarrow \coprod_{\substack{d \in \mathbf{Z} \\ d \text { odd }}}\left(W_{n-\frac{1}{2} d(d-1)}\right)^{\wedge}
$$

(b) Let $G=S O_{N}$ with $p \neq 2$. Then $(L, C, \mathcal{E}) \in \mathcal{M}_{G}$ if and only if $L$ is of type $B_{m}$ (resp. $\left.D_{m}\right)$ for some $m$ such that $m=\frac{1}{2}\left(d^{2}-1\right)\left(\right.$ resp. $\left.m=\frac{1}{2} d^{2}\right)$ and that $d \equiv N(\bmod 2)$ in the case where $N$ is odd (resp. $N$ is even) with $m \geq 0$. We have $N_{G}(L) / L \simeq W_{\left(N-d^{2}\right) / 2}$ if $m \geq 1$, and $N_{G}(L) / L \simeq W_{n}$ (resp. $W_{n}^{\prime}$ ) if $m=0$, namely if $L$ is a maximal torus $T$, in the case where $N$ is odd (resp. $N$ is even). Thus the generalized Springer correspondence is given by a bijection

$$
\begin{aligned}
& \mathcal{N}_{G} \longleftrightarrow \coprod_{\substack{d \geq 1 \\
d \text { odd }}}\left(W_{\left(N-d^{2}\right) / 2}\right)^{\wedge} \quad(N \text { : odd }) \\
& \mathcal{N}_{G} \longleftrightarrow W_{n}^{\prime} \coprod\left(\coprod_{\substack{d>0 \\
d \text { even }}}\left(W_{\left(N-d^{2}\right) / 2}\right)^{\wedge}\right) \quad(N \text { : even })
\end{aligned}
$$

(c) Let $G=S p_{2 n}$ with $p=2$. Then $(L, C, \mathcal{E}) \in \mathcal{M}_{G}$ if and only if $L$ is of type $C_{m}$ for some $m$ of the form $m=d(d-1)$ with $d \geq 1$. We have $N_{G}(L) / L \simeq W_{n-d(d-1)}$. Hence as in the case (a), the generalized Springer correspondence is given by

$$
\mathcal{N}_{G} \longleftrightarrow \coprod_{\substack{d \in \mathbf{Z} \\ d \text { odd }}}\left(W_{n-d(d-1)}\right)^{\wedge}
$$


(d) Let $G=S O_{2 n}$ with $p=2$. Then $(L, C, \mathcal{E}) \in \mathcal{M}_{G}$ if and only if $L$ is of type $D_{m}$ for some $m$ of the form $m=d^{2}$ with $d \geq 0$, even. We have $N_{G}(L) / L \simeq W_{n-d^{2}}$ if $d \geq 1$ and $N_{G}(L) / L \simeq W_{n}^{\prime}$ if $d=0$, namely if $L$ is a maximal torus of $G$. Hence the generalized Springer correspondence is given by

$$
\mathcal{N}_{G} \longleftrightarrow W_{n}^{\prime} \coprod\left(\coprod_{\substack{d>0 \\ d \text { even }}}\left(W_{n-d^{2}}\right)^{\wedge}\right)
$$

3.2. The generalized Springer correspondence for classical groups is described in terms of symbols. We review the notion of symbols following [L1], [LS]. Let $r, s \in \mathbf{Z}_{\geq 1}, d \in \mathbf{Z}$. For each integer $n \geq 1$ let $\widetilde{X}_{n, d}^{r, s}$ be the set of all ordered pairs $(A, B)$, where $A=\left\{a_{1}, \ldots, a_{m+d}\right\}, B=\left\{b_{1}, \ldots, b_{m}\right\}$ (for some $m$ ) are subsets of $\mathbf{Z}_{\geq 0}$ satisfying the following conditions.

$$
\begin{aligned}
a_{i}-a_{i-1} & \geq r+s \quad(1<i \leq m+d), \\
b_{i}-b_{i-1} & \geq r+s \quad(1<i \leq m), \\
b_{1} & \geq s, \\
\sum a_{i}+\sum b_{i} & =n+(r+s)(m+[d / 2])(m+d-[d / 2])-r(m+[d / 2]) .
\end{aligned}
$$

(In the case where $r+s=0, A$ or $B$ contains elements with multiplicities. In that case, we regard it as a sequence of integers.)

Note that if $r=s=1$ and $d$ is odd the fourth condition is written as

$$
\sum a_{i}+\sum b_{i}=n+\frac{1}{2}(2 m+d)(2 m+d-1),
$$

and if $r=2, s=0$ it is written as

$$
\sum a_{i}+\sum b_{i}=n+\frac{1}{2}\left((2 m+d-1)^{2}-1\right)
$$

Let $X_{n, d}^{r, s}$ be the set of equivalence classes on $\widetilde{X}_{n, d}^{r, s}$ for the equivalence relation generated by

$$
(A, B) \sim(\{0\} \cup(A+r+s),\{s\} \cup(B+r+s)),
$$

where $A+r+s$ denotes the set $\left\{a_{1}+(r+s), \ldots, a_{m+d}+(r+s)\right\}$ and so on for $B$. We put

$$
X_{n}^{r, s}=\coprod_{d \text { odd }} X_{n, d}^{r, s}
$$


An element in $X_{n, d}^{r, s}$ is called an $(r, s)$-symbol of rank $n$ and defect $d$, which we also denote by $(A, B)$.

We consider the special case where $s=0$. In that case, $(A, B) \mapsto(B, A)$ defines a bijection from $X_{n, d}^{r, 0}$ to $X_{n,-d}^{r, 0}$ and so induces an involution of each of the following sets,

$$
X_{n, \text { even }}^{r, 0}=\coprod_{d \text { even }} X_{n, d}^{r, 0}, \quad X_{n, \text { odd }}^{r, 0}=\coprod_{d \text { odd }} X_{n, d}^{r, 0} .
$$

Let $Y_{n \text {, odd }}^{r}$ (resp. $Y_{n \text {, even }}^{r}$ ) be the set obtained as the quotient of $X_{n, \text { odd }}^{r, 0}$ (resp. $\left.X_{n, \text { even }}^{r, 0}\right)$ by this involution, with the convention that the symbol invariant by the involution, i.e., the symbol $(A, A)$ which we call the degenerate symbol, is counted twice. For $d \geq 0$, the image of $X_{n, d}^{r, 0}$ in $Y_{n \text {, odd }}^{r}$ or $Y_{n \text {, even }}^{r}$ is denoted by $Y_{n, d}^{r}$. One can regard the element in $Y_{n, d}^{r}$ as a symbol $(A, B)$ in $X_{n, d}^{r, 0}$ considered as an unordered pair.

3.3. The set $W_{n}^{\wedge}$ is parametrized by ordered pairs of partitions $(\alpha, \beta)$ such that $|\alpha|+|\beta|=n$. For a fixed $d \in \mathbf{Z}$, one can express the partitions $\alpha, \beta$ as $\alpha: \alpha_{1} \leq \alpha_{2} \leq \cdots \leq \alpha_{m+d}, \beta: \beta_{1} \leq \beta_{2} \leq \cdots \leq \beta_{m}$ for a suitable $m$, by allowing 0 in the entries. Then $(A, B) \in \widetilde{X}_{n, d}^{0,0}$ for $A=\left\{\alpha_{1}, \ldots, \alpha_{m+d}\right\}$, $B=\left\{\beta_{1}, \ldots, \beta_{m}\right\}$, and this induces a well-defined bijection between $W_{n}^{\wedge}$ and $X_{n, d}^{0,0}$. The same map induces a bijection between $W_{n}^{\wedge}$ and $Y_{n, d}^{0}$ if $d \geq 1$. On the other hand, the set $\left(W_{n}^{\prime}\right)^{\wedge}$ is parametrized by unordered pairs of partitions $(\alpha, \beta)$ such that $|\alpha|+|\beta|=n$, under the convention that $(\alpha, \alpha)$ is counted twice. Thus in a similar way as above, we have a natural bijection between $\left(W_{n}^{\prime}\right)^{\wedge}$ and $Y_{n, 0}^{0}$.

For a given $r, s, d$, we define a symbol $\Lambda_{d}^{r, s}=(A, B)$ as follows.

$$
\begin{cases}A=\{0,(r+s), \ldots,(d-1)(r+s)\}, \quad B=\emptyset & \text { if } d>0, \\ A=\emptyset, \quad B=\{s, s+(r+s), \ldots, s+(-d-1)(r+s)\} & \text { if } d<0, \\ A=\emptyset, \quad B=\emptyset & \text { if } d=0 .\end{cases}
$$

It is easy to see that $\Lambda_{d}^{r, s} \in X_{n_{0}, d}^{r, s}$ with $n_{0}=(r+s)[d / 2](d-[d / 2])-s[d / 2]$, and that the set $X_{n_{0}, d}^{r, s}$ consists of a unique element $\Lambda_{d}^{r, s}$. In the case where $s=0$, let $\Lambda_{d}^{r}$ be the image of $\Lambda_{d}^{r, 0}$ under the map $X_{n_{0}, d}^{r, 0} \rightarrow Y_{n_{0}, d}^{r}$. For each $d \in \mathbf{Z}$, one can define a map

$$
X_{n-n_{0}, d}^{0,0} \longrightarrow X_{n, d}^{r, s}, \quad \Lambda \longmapsto \Lambda+\Lambda_{d}^{r, s}
$$


which gives a bijection $X_{n-n_{0}, d}^{r, s} \simeq X_{n, d}^{r, s}$. (Note, in general, that the sum of two symbols $\Lambda, \Lambda^{\prime}$ with the same defect is defined by choosing representatives $\Lambda=(A, B), \Lambda^{\prime}=\left(A^{\prime}, B^{\prime}\right)$ such that $|A|+|B|=\left|A^{\prime}\right|+\left|B^{\prime}\right|$, namely of the same shape, and then by adding entry-wise.) Similarly for each $d \geq 0$, one can define a bijection

$$
Y_{n-n_{0}, d}^{0} \longrightarrow Y_{n, d}^{r}, \quad \Lambda \longmapsto \Lambda+\Lambda_{d}^{r}
$$

Combining (3.3.1) with the bijection $W_{n-n_{0}}^{\wedge} \simeq X_{n-n_{0}, d}^{0,0}$ above, we have a bijection

$$
W_{n-n_{0}}^{\wedge} \longrightarrow X_{n, d}^{r, s}
$$

Similarly, combining (3.3.2) with the bijections $W_{n-n_{0}}^{\wedge} \simeq Y_{n-n_{0}, d}^{0}$ for $d>0$ and $\left(W_{n-n_{0}}^{\prime}\right)^{\wedge} \simeq Y_{n-n_{0}, 0}^{0}$, we have bijections

$$
\begin{array}{cc}
W_{n-n_{0}}^{\wedge} \longrightarrow Y_{n, d}^{r} \quad(d>0), \\
\left(W_{n-n_{0}}^{\prime}\right)^{\wedge} \longrightarrow Y_{n, d}^{r} \quad(d=0) .
\end{array}
$$

3.4. A symbol $(A, B) \in X_{n, d}^{r, s}$ is said to be distinguished if $d=0$ or 1 , and

$$
\begin{array}{ll}
a_{1} \leq b_{1} \leq a_{2} \leq b_{2} \leq \cdots \leq a_{m} \leq b_{m} & (d=0), \\
a_{1} \leq b_{1} \leq a_{2} \leq b_{2} \leq \cdots \leq a_{m} \leq b_{m} \leq a_{m+1} & (d=1) .
\end{array}
$$

A symbol $(A, B) \in Y_{n, d}^{r}$ for $d \geq 0$ is said to be distinguished if it is an image of a distinguished symbol in $X_{n, d}^{r, 0}$.

Assume that $r \geq 1$. The two symbols $(A, B),\left(A^{\prime}, B^{\prime}\right) \in X_{n}^{r, s}$ are said to be similar if $A \cup B=A^{\prime} \cup B^{\prime}, A \cap B=A^{\prime} \cap B^{\prime}$, namely under some shift, $A \cup B$ coincides with $A^{\prime} \cup B^{\prime}$ with multiplicities. This defines an equivalence relation on the set $X_{n}^{r, s}$, and an equivalence class is called a similarity class in $X_{n}^{r, s}$. A similarity class in $Y_{n \text {, even }}^{r}$ or $Y_{n \text {, odd }}^{r}$ is defined in a similar way. A similarity class in $Y_{n \text {, even }}^{r}$ containing $(A, A)$ is called a degenerate class, which consists of two copies of $(A, A)$. It is easy to see that each (non-degenerate) similarity class contains a unique distinguished symbol.

It is known by [L1], [LS] that a similarity class in $X_{n}^{r, s}, Y_{n, \text { even }}^{r}$ or $Y_{n \text {, odd }}^{r}$ is in a natural way regarded as a vector space over $\mathbf{F}_{2}$ as follows. Let $\Lambda=(A, B)$ be a distinguished symbol in a similarity class $\mathcal{C}$ in $X_{n}^{r, s}, Y_{n, \text { even }}^{r}$ or $Y_{n, \text { odd }}^{r}$. We assume that $A \neq B$ if $(A, B) \in Y_{n, \text { even }}^{r}$, and put $S=(A \cup$ 
$B) \backslash(A \cap B)$. Then $S \neq \emptyset$ and it is written as $S=\left\{c_{1}, c_{2}, \ldots, c_{t}\right\}$ in an increasing order. A non-empty subset $I=\left\{c_{i}, c_{i+1}, \ldots, c_{j}\right\}$ of $S$ is called an interval if $c_{k+1}-c_{k}<r+s$ for $i \leq k<j$ and it is maximal with respect to this condition. We say that $c_{i}$ is the tail of $I$. An interval is called an initial interval if $c_{i}<s$. Hence the initial interval exists only in the case where $s>0$, and in that case, it exists uniquely after some shift. $S$ is a disjoint union of intervals.

Assume that $\mathcal{C} \subset X_{n}^{r, s}$. Let $I$ be an interval which is not initial with the tail $c$. If $c \in A$ (resp. $c \in B$ ), then there exists a unique $\left(A^{\prime}, B^{\prime}\right) \in \mathcal{C}$ such that $c \in B$ (resp. $c \in A$ ) and that $A \cap J=A^{\prime} \cap J, B \cap J=B^{\prime} \cap J$ for all other intervals $J$. This means that $\left(A^{\prime}, B^{\prime}\right)$ is obtained from $(A, B)$ by permuting the entries in the interval $I$. All the symbols in $\mathcal{C}$ are obtained from $(A, B)$ by permuting the entries in certain intervals. Let $\mathcal{I}$ be the set of non-initial intervals in $S$ and $\mathcal{P}(\mathcal{I})$ the set of all subsets of $\mathcal{I}$. The above argument shows that $\mathcal{C}$ is in bijection with the set $\mathcal{P}(\mathcal{I})$, which has a natural structure of $\mathbf{F}_{2}$ vector space with origin $\Lambda$ and is denoted by $V_{\Lambda}^{r, s}$. In the case where $\mathcal{C} \subset Y_{n \text {, even }}^{r}$ or $\mathcal{C} \subset Y_{n \text {,odd }}^{r}(\mathcal{C}:$ non-degenerate $), \mathcal{C}$ is in bijection with the quotient set of $\mathcal{P}(\mathcal{I})$ under the relation $\mathcal{K} \sim \mathcal{I} \backslash \mathcal{K}$ for $\mathcal{K} \in \mathcal{P}(\mathcal{I})$. Hence $\mathcal{C}$ is identified with the $\mathbf{F}_{2}$ vector space $V_{\Lambda}^{r, 0} / L$, where $L$ is a line generated by $\mathcal{I} \in \mathcal{P}(\mathcal{I})$, which we denote by $V_{\Lambda}^{r}$.

3.5. Let $G$ be as in 3.1. We associate the sets $X_{n}^{1,1}, Y_{n, \text { even }}^{2}, Y_{n \text {, odd }}^{2}$ for $S p_{2 n}, S O_{2 n}, S O_{2 n+1}$ with $p \neq 2$ and $X_{n}^{2,2}, Y_{n, \text { even }}^{4}$ for $S p_{2 n}, S O_{2 n}$ with $p=2$. Recall $n_{0}$ in 3.3 .

(a) The case $X_{n}^{1,1}$. We have $r=s=1$, and $n_{0}=\frac{1}{2} d(d-1)$ for odd $d$. Hence (3.3.3) implies a bijection

$$
X_{n}^{1,1} \longleftrightarrow \coprod_{\substack{d \in \mathbf{Z} \\ d \text { odd }}}\left(W_{n-\frac{1}{2} d(d-1)}\right)^{\wedge}
$$

(b) The case $Y_{n \text {, even }}^{2}$ or $Y_{n \text {, odd }}^{2}$. We have $r=2, s=0$, and $n_{0}=\frac{1}{2} d^{2}$ for even $d$ and $n_{0}=\frac{1}{2}\left(d^{2}-1\right)$ for odd $d$. Hence (3.3.4) implies bijections

$$
\begin{aligned}
& Y_{n, \text { odd }}^{2} \longleftrightarrow \coprod_{\substack{d \geq 1 \\
d \text { odd }}}\left(W_{n-\frac{1}{2}\left(d^{2}-1\right)}\right)^{\wedge}, \\
& Y_{n, \text { even }}^{2} \longleftrightarrow\left(W_{n}^{\prime}\right)^{\wedge} \coprod\left(\coprod_{\substack{d>0 \\
d \text { even }}}\left(W_{n-\frac{1}{2} d^{2}}\right)^{\wedge}\right) .
\end{aligned}
$$


(c) The case $X_{n}^{2,2}$. We have $r=s=2$, and $n_{0}=d(d-1)$ for odd $d$. Hence (3.3.3) implies a bijection

$$
X_{n}^{2,2} \longleftrightarrow \coprod_{\substack{d \in \mathbf{Z} \\ d \text { odd }}}\left(W_{n-d(d-1)}\right)^{\wedge}
$$

(d) The case $Y_{n \text {, even }}^{4}$. We have $r=4, s=0$, and $n_{0}=d^{2}$ for even $d$. Hence (3.3.4) implies a bijection

$$
Y_{n, \text { even }}^{4} \longleftrightarrow\left(W_{n}^{\prime}\right)^{\wedge} \coprod\left(\coprod_{\substack{d>0 \\ d \text { even }}}\left(W_{n-d^{2}}\right)^{\wedge}\right) .
$$

3.6. Let $X=X_{n}^{1,1}, Y_{n \text {, odd }}^{2}, Y_{n \text {, even }}^{2}$ or $X_{n}^{2,2}, Y_{n, \text { even }}^{4}$ according as $G=$ $S p_{2 n}, S O_{2 n+1}, S O_{2 n}$ with $p \neq 2$, or $G=S p_{2 n}, S O_{2 n}$ with $p=2$. In view of the bijections in 3.5 and the discussion in 3.1, the generalized Springer correspondence can be described by giving a bijection between $\mathcal{N}_{G}$ and $X$. By [L1], [LS], this bijection is given explicitly in such a way that the set $G_{\text {uni }} / \sim$ of unipotent classes in $G$ is in bijection with the set $X / \sim$ of similarity classes in $X$. In what follows, we define a map $\rho: G_{\text {uni }} / \sim \rightarrow X / \sim$ by associating a distinguished symbol $\Lambda=\rho(C) \in X$ for each unipotent class $C$ in $G$.

(a) $G=S p_{2 n}$ with $p \neq 2$. Let $C_{\lambda}$ be a unipotent class of $G$ as in 2.2 , where $\lambda$ is a partition of $2 n$. We express $\lambda$ as $\lambda_{1} \leq \lambda_{2} \leq \cdots \leq$ $\lambda_{2 m}$ for some $m$, by allowing 0 in the entries if necessary. We divide the sequence $\left\{\lambda_{1}, \lambda_{2}, \ldots, \lambda_{2 m}\right\}$ into the union of blocks as follows. If $\lambda_{i}$ is even, let $\left\{\lambda_{i}\right\}$ be a block. If $\lambda_{i}=h$ is odd, the sequence $A_{h}=\left\{\lambda_{k} \mid \lambda_{k}=h\right\}$ consisting of even elements, which we write as $\left\{\lambda_{a}, \lambda_{a+1}, \ldots, \lambda_{b}\right\}$ for some $b>a$. Then we divide $A_{h}$ into a disjoint union of two elements blocks $\left\{\lambda_{a}, \lambda_{a+1}\right\} \cup\left\{\lambda_{a+2}, \lambda_{a+3}\right\} \cup \cdots \cup\left\{\lambda_{b-1}, \lambda_{b}\right\}$. We define a sequence $\nu_{1}, \ldots, \nu_{2 m}$ as follows. Put

$$
\begin{cases}\nu_{i}=\lambda_{i} / 2+i & \text { if }\left\{\lambda_{i}\right\} \text { is a block, } \\ \nu_{i}=\nu_{i+1}=\left(\lambda_{i}+1\right) / 2+i & \text { if }\left\{\lambda_{i}, \lambda_{i+1}\right\} \text { is a block, }\end{cases}
$$

and put $A=\left\{0, \nu_{2}, \nu_{4}, \ldots, \nu_{2 m}\right\}, B=\left\{\nu_{1}, \nu_{3}, \ldots, \nu_{2 m-1}\right\}$. Then $\Lambda=(A, B)$ gives rise to a distinguished symbol in $X_{n}^{1,1}$, which is independent of the choice of $m$, and $C_{\lambda} \mapsto \Lambda$ gives the required bijection $\rho$. Actually, the map 
$\rho$ was defined in $[\mathrm{L} 1,11.6]$. Although the definition given there is not the same as ours, it is easily checked that they coincide with each other.

(b) $G=S O_{N}$ with $p \neq 2$. Let $C_{\lambda}$ be a unipotent class of $G$ as in 2.2, where $\lambda$ is a partition of $N$. We choose $M$ such that $M \equiv N$ (mod 2) and express $\lambda$ as $\lambda_{1} \leq \lambda_{2} \leq \cdots \leq \lambda_{M}$. We divide the sequence $\left\{\lambda_{1}, \lambda_{2}, \ldots, \lambda_{M}\right\}$ into the union of blocks as follows. If $\lambda_{i}$ is odd, let $\left\{\lambda_{i}\right\}$ be a block. If $\lambda_{i}=h$ is even, the sequence $A_{h}=\left\{\lambda_{k} \mid \lambda_{k}=h\right\}$ consists of even elements. As in the case (a), we divide $A_{h}$ as a disjoint union of two elements blocks, $\left\{\lambda_{a}, \lambda_{a+1}\right\} \cup\left\{\lambda_{a+2}, \lambda_{a+3}\right\} \cup \cdots \cup\left\{\lambda_{b-1}, \lambda_{b}\right\}$. We define a sequence $\nu_{1}, \nu_{2}, \ldots, \nu_{M}$ as follows. Put

$$
\begin{cases}\nu_{i}=\left(\lambda_{i}-3\right) / 2+i & \text { if }\left\{\lambda_{i}\right\} \text { is a block, } \\ \nu_{i}=\nu_{i+1}=\left(\lambda_{i}-2\right) / 2+i & \text { if }\left\{\lambda_{i}, \lambda_{i+1}\right\} \text { is a block, }\end{cases}
$$

and put $A=\left\{\nu_{1}, \nu_{3}, \ldots, \nu_{[(M+1) / 2]}\right\}, B=\left\{\nu_{2}, \nu_{4}, \ldots, \nu_{[M / 2]}\right\}$. Then $\Lambda=$ $(A, B)$ gives rise to a distinguished symbol in $Y_{n \text {,odd }}^{2}$ or $Y_{n \text {, even according }}^{2}$ as $N$ is odd or even, which is independent of the choice of $M$. The map $C_{\lambda} \mapsto \Lambda$ gives the required bijection $\rho$. The proof follows, as in the case (a), from the discussion in $[\mathrm{L} 1,11.7]$.

(c) $G=S p_{2 n}$ with $p=2$. The map $\rho$ is defined in [LS, 2.1]. The following definition is slightly modified from the original one so as to fit to the case (a). Let $C_{\lambda, \varepsilon}$ be a unipotent class of $G$ as in 2.3, where $\lambda$ is a partition of $2 n$. Here $\lambda$ is the same as in the case (a), and we express it as $\lambda_{1} \leq \cdots \leq \lambda_{2 m}$ for some $m$. We use the convention that $\varepsilon(0)=1$. We divide the set $\left\{\lambda_{1}, \lambda_{2}, \ldots, \lambda_{2 m}\right\}$ into a disjoint union of blocks as follows. If $\varepsilon\left(\lambda_{i}\right)=1$, then $\left\{\lambda_{i}\right\}$ is a block. If $\varepsilon\left(\lambda_{i}\right)=0$ or $\omega$ for $\lambda_{i}=h$, the sequence $A_{h}=\left\{\lambda_{k} \mid \lambda_{k}=h\right\}$ has even cardinality, and it is divided into blocks as in the case (a). We define a sequence $\nu_{1}, \ldots, \nu_{2 m}$ as follows.

(i) If $\left\{\lambda_{i}\right\}$ is a block, put

$$
\nu_{i}=\lambda_{i} / 2+2 i .
$$

(ii) If $\left\{\lambda_{i}, \lambda_{i+1}\right\}$ is a block and $\varepsilon\left(\lambda_{i}\right)=\omega$, put

$$
\begin{aligned}
& \nu_{i}=\left(\lambda_{i}+1\right) / 2+2 i, \\
& \nu_{i+1}=\nu_{i}+1 .
\end{aligned}
$$

(iii) If $\left\{\lambda_{i}, \lambda_{i+1}\right\}$ is a block and $\varepsilon\left(\lambda_{i}\right)=0$, put

$$
\begin{aligned}
& \nu_{i}=\left(\lambda_{i}+2\right) / 2+2 i, \\
& \nu_{i+1}=\nu_{i} .
\end{aligned}
$$


Put $A=\left\{0, \nu_{2}, \nu_{4}, \ldots, \nu_{2 m}\right\}, B=\left\{\nu_{1}, \nu_{3}, \ldots, \nu_{2 m-1}\right\}$. Then by [LS, 2.2], $\Lambda=(A, B)$ gives rise to a distinguished symbol in $X_{n}^{2,2}$, which is independent of the choice $m$, and $C_{\lambda, \varepsilon} \mapsto \Lambda$ gives the required bijection

(d) $G=S O_{2 n}$ with $p=2$. The map $\rho$ is defined in [LS, 3.1]. Let $C_{\lambda, \varepsilon}$ be a unipotent class in $G$. $\lambda$ is the same as in the case (c), and we define the block in the same way as the case (c). However, here we use the convention that $\varepsilon(0)=0$. Note that in the sequence $\left\{\lambda_{1}, \ldots, \lambda_{2 m}\right\}$ the multiplicity of 0 is even since the number of non-zero $\lambda_{i}$ is even (cf. 2.3). We define a sequence $\nu_{1}, \ldots, \nu_{2 m}$ as follows.

(i) If $\left\{\lambda_{i}\right\}$ is a block, put

$$
\nu_{i}=\left(\lambda_{i}-6\right) / 2+2 i .
$$

(ii) If $\left\{\lambda_{i}, \lambda_{i+1}\right\}$ is a block and $\varepsilon\left(\lambda_{i}\right)=\omega$, put

$$
\begin{aligned}
& \nu_{i}=\left(\lambda_{i}-5\right) / 2+2 i, \\
& \nu_{i+1}=\nu_{i}+1 .
\end{aligned}
$$

(iii) If $\left\{\lambda_{i}, \lambda_{i+1}\right\}$ is a block and $\varepsilon\left(\lambda_{i}\right)=0$, put

$$
\begin{aligned}
& \nu_{i}=\left(\lambda_{i}-4\right) / 2+2 i, \\
& \nu_{i+1}=\nu_{i} .
\end{aligned}
$$

Put $A=\left\{\nu_{1}, \nu_{3}, \ldots, \nu_{2 m-1}\right\}, B=\left\{\nu_{2}, \nu_{4}, \ldots, \nu_{2 m}\right\}$. Then by [LS, 3.2], $\Lambda=$ $(A, B)$ gives rise to a distinguished symbol in $Y_{n, \text { even }}^{4}$, which is independent of the choice of $m$, and $C_{\lambda, \varepsilon} \mapsto \Lambda$ gives the required bijection.

3.7. We return to the setting in the beginning of 3.6, and let $\rho$ : $G_{\text {uni }} / \sim \rightarrow X / \sim$ be the bijection constructed in 3.6. By making use of $\rho$, we shall construct a bijection $\widetilde{\rho}: \mathcal{N}_{G} \rightarrow X$. For a unipotent class $C$ in $G$, let $\mathcal{C}$ be the similarity class in $X$ containing the distinguished symbol $\Lambda=\rho(C)$. Take $u \in C$. As discussed in $3.4, \mathcal{C}$ has a natural structure of $\mathbf{F}_{2}$-vector space $V_{A}^{r, s}$ for $X=X_{n}^{1,1}$ or $X_{n}^{2,2}$ with the basis corresponding to the set of intervals. Since $A_{G}(u)$ is an elementary abelian 2-group, it has a natural structure of $\mathbf{F}_{2^{-}}$-vector space, and so does the dual group $A_{G}(u)^{\wedge}$. It was shown in $[\mathrm{L} 1,11],[\mathrm{LS}, 2.2]$ that $V_{\Lambda}^{r, s}$ is naturally identified with $A_{G}(u)^{\wedge}$, where the set of intervals is in bijection with the set of generators in $A_{G}(u)$ given in 2.4; if $I$ is an interval corresponding to the generator $a_{i}$ of $A_{G}(u)$, we associate the character $\chi_{i}$ of $A_{G}(u)$ which takes the value -1 
for $a_{i}$ and 1 for other generators. Similar argument also works for the case where $X=Y_{n \text {,odd }}^{2}, Y_{n \text {, even }}^{2}$ and $Y_{n \text {, even }}^{4}$, and by [L1, 11], [LS, 3.2] $V_{A}^{r}$ is naturally identified with $A_{G}(u)^{\wedge}$. Hence the similarity class $\mathcal{C}$ can be identified with $A_{G}(u)^{\wedge}$.

On the other hand, for a fixed $C, G$-equivariant simple local systems on $C$ are parametrized by $A_{G}(u)^{\wedge}$. Thus combining with the above argument, we obtain a bijection $\widetilde{\rho}: \mathcal{N}_{G} \rightarrow X$. This bijection describes combinatorially the generalized Springer correspondence, namely,

Theorem 3.8. ([L1, 12.3, 13.3], [LS, 2.4, 3.3]) Let $G$ be as in 3.1. Then the composite of $\widetilde{\rho}$ with the bijection in 3.5 gives the generalized Springer correspondence in 3.1 .

\section{$\S 4$. Main results}

4.1. Let $G$ be as in 2.1 and we apply the argument in Section 1 for $G$. First consider the case where $G$ is of split type. For each unipotent class $C^{\prime}$ in $G$, we choose a split element $u \in C^{\prime F}$ described in Section 2, i.e., (2.9.3) for $G=S p_{2 n}$ or $S O_{N}$ with $p \neq 2$, (2.7.3) for $G=S p_{2 n}$ with $p=2$, (2.8.1) for $G=S O_{N}$ with $p=2$. For each pair $\left(C^{\prime}, \mathcal{E}^{\prime}\right) \in \mathcal{N}_{G}^{F}$, we fix an isomorphism $\psi_{0}=\psi_{\mathcal{E}^{\prime}}: F^{*} \mathcal{E}^{\prime} \rightarrow \mathcal{E}^{\prime}$ in 1.6 so that the induced isomorphism $\mathcal{E}_{u}^{\prime} \rightarrow \mathcal{E}_{u}^{\prime}$ is identity. (Note that $F$ acts trivially on $A_{G}(u)$. This is known by [SS] for the case where $p \neq 2$, and follows from (2.7.4) for the case where $p=2$.) Take $(L, C, \mathcal{E}) \in \mathcal{M}_{G}^{F}$. Then $L$ is of the same type as $G$ of split type. Thus we choose the split element $u_{0} \in C^{F}$. We fix an isomorphism $\varphi_{0}: F^{*} \mathcal{E} \rightarrow \mathcal{E}$ in 1.6 so that the induced isomorphism $\mathcal{E}_{u_{0}} \rightarrow \mathcal{E}_{u_{0}}$ is identity. Let $V_{\left(C^{\prime}, \mathcal{E}^{\prime}\right)}$ be the irreducible $\mathcal{W}$-module and $\sigma_{\left(C^{\prime}, \mathcal{E}^{\prime}\right)}$ be the isomorphism on $V_{\left(C^{\prime}, \mathcal{E}^{\prime}\right)}$ given in 1.6. Since $F$ is of split type, $F$ acts trivially on $\mathcal{W}$ and so $\sigma_{\left(C^{\prime}, \mathcal{E}^{\prime}\right)}$ commutes with the action of $\mathcal{W}$. It follows that $\sigma_{\left(C^{\prime}, \mathcal{E}^{\prime}\right)}$ is a scalar map.

Next consider the case where $G=S O_{2 n}$ with $F=F_{0} \sigma$ of non-split type. We choose the split elements $u^{\prime} \in C^{\prime F}$ and $u_{0}^{\prime} \in C^{F}$ as in 2.10, and fix $\psi_{0}=\psi_{\mathcal{E}^{\prime}}, \varphi_{0}$ as above. (Again $F$ acts trivially on $A_{G}\left(u^{\prime}\right)$ by (2.10.2).) Let $(L, C, \mathcal{E}) \in \mathcal{M}_{G}^{F}$. If $L \neq T, \mathcal{W}$ is a Weyl group of type $B$ and so $F$ acts trivially on $\mathcal{W}$. Hence $\sigma_{\left(C^{\prime}, \mathcal{E}^{\prime}\right)}$ is a scalar map. While if $L=T, \mathcal{W}=W_{n}^{\prime}$ is the Weyl group of type $D_{n}$ and $F$ acts non-trivially on $\mathcal{W}$. Note that $\sigma$ acts on $W_{n}^{\prime}$ and the semidirect product $W_{n}^{\prime} \rtimes\langle\sigma\rangle$ is isomorphic to $W_{n}$. Assume that $V_{\left(C^{\prime}, \mathcal{E}^{\prime}\right)}=E$ is $F$-stable. Then $E$ can be extended to an irreducible representation $\widetilde{E}$ of $W_{n}$ via the map $\sigma_{\left(C^{\prime}, \mathcal{E}^{\prime}\right)}$. Since $\sigma^{-1} \circ \sigma_{\left(C^{\prime}, \mathcal{E}^{\prime}\right)}$ commutes 
with the action of $\mathcal{W}$, it acts as a scalar on $E$. Thus in order to describe the map $\sigma_{\left(C^{\prime}, \mathcal{E}^{\prime}\right)}$, we have only to determine this scalar together with the representation $\widetilde{E}$.

We recall the preferred extension $\widetilde{E}$ of $W_{n}$ due to [L2, IV, 17.2]. An $F$ stable irreducible representation $E$ of $W_{n}^{\prime}$ is parametrized by an unordered pair $(\alpha ; \beta)$ of partitions such that $|\alpha|+|\beta|=n$ and that $\alpha \neq \beta$. We write $\alpha: \alpha_{1} \leq \cdots \leq \alpha_{m}, \beta: \beta_{1} \leq \cdots \leq \beta_{m}$, and define a symbol $\Lambda_{E}$ associated to $E$ by an unordered pair $(\lambda ; \mu)$ with $\lambda_{i}=\alpha_{i}+i-1, \mu_{j}=\beta_{j}+j-1$. (This is a different type of symbols from those appeared in Section 3.) Irreducible representations of $W_{n}$ are parametrized in a similar way, but by using an ordered pair $(\alpha ; \beta)$ and its associated symbol $(\lambda ; \mu)$. For an $F$-stable irreducible representation $E$, there exists two extensions to $W_{n}$, which correspond to two symbols $(\lambda ; \mu)$ and $(\mu ; \lambda)$ for $W_{n}$. An extension $\widetilde{E}$ of $E$ is called the preferred extension of $E$ if in the symbol $\Lambda_{\widetilde{E}}$, the smallest number which does not appear in both entries appears in the second entry. For example, $(n ; 0)$ is the symbol associated to the unit representation of $W_{n}^{\prime}$, and it is extended to the unit representation $(n ; 0)$ or the long sigh representation $(0 ; n)$ of $W_{n}$. In this case, $(n ; 0)$ is the preferred extension.

We can state our main results.

TheOREM 4.2. Let $G=S p_{2 n}, S O_{N}$ with $F$ of split type ( $N$ is even if $p=2)$. Then $\sigma_{\left(C^{\prime}, \mathcal{E}^{\prime}\right)}$ is $q^{\left(a_{0}+r\right) / 2}$ times identity.

TheOREM 4.3. Let $G=S O_{2 n}$ with $F$ of non-split type.

(i) Suppose $L \neq T$. Then $\sigma_{\left(C^{\prime}, \mathcal{E}^{\prime}\right)}$ is $q^{\left(a_{0}+r\right) / 2}$ times identity.

(ii) Suppose $L=T$. Then $\sigma_{C^{\prime}, \mathcal{E}^{\prime}}=q^{\left(a_{0}+r\right) / 2} \sigma$, and $W_{n}^{\prime}\langle\sigma\rangle$-module $\widetilde{E}$ coincides with the preferred extension of $E$.

In view of Lemma 1.11, we have the following corollary.

Corollary 4.4. Let $G$ be as in 2.1. For each $\left(C^{\prime}, \mathcal{E}^{\prime}\right) \in \mathcal{N}_{G}^{F}$, choose $\psi_{0}: F^{*} \mathcal{E}^{\prime} \stackrel{\sim}{\rightarrow} \mathcal{E}^{\prime}$ by choosing a split element $u \in C^{\prime F}$. Let $\gamma$ be the constant given in 1.10 . Then we have $\gamma=1$, namely we have $Y_{j}=Y_{j}^{0}$ for $j=\left(C^{\prime}, \mathcal{E}^{\prime}\right)$.

4.5. We prove the theorem by making use of the restriction formula in Corollary 1.9. We choose a standard parabolic subgroup $Q=M U_{Q}$ such that the Levi subgroup $M$ of $Q$ is of the same type as $G$ with semisimple rank $n-1$. Take $(L, C, \mathcal{E}) \in \mathcal{M}_{G}^{F}$ and let $P$ be the $F$-stable standard 
parabolic subgroup of $G$ whose Levi subgroup is $L$. Then we have $P \subset Q$ and $L \subset M$. Take $\left(C^{\prime}, \mathcal{E}^{\prime}\right) \in \mathcal{N}_{G}^{F}$, and take a split element $u \in C^{\prime F}$. Also take $\left(C_{1}, \mathcal{E}_{1}\right) \in \mathcal{N}_{M}^{F}$, and choose a split element $v \in C_{1}^{F}$. Let $\rho$ (resp. $\rho_{1}$ ) be the irreducible representation of $A_{G}(u)$ (resp. $A_{M}(v)$ ) corresponding to $\mathcal{E}^{\prime}$ (resp. $\mathcal{E}_{1}$ ). Since $F$ acts trivially on $A_{G}(u)$ and $A_{M}(v)$, the extension $\widetilde{\rho \otimes \rho_{1}^{*}}$ to $\widetilde{A}(u, v)$ in 1.8 is just the trivial extension of $\rho \otimes \rho_{1}^{*}$. Then we have the following lemma.

Lemma 4.6. Assume that $F$ acts trivially on $\mathcal{W}$ and on $\mathcal{W}_{1}$. Let $E \in$ $\mathcal{W}^{\wedge}\left(\right.$ resp. $\left.E_{1} \in \mathcal{W}_{1}^{\wedge}\right)$ be corresponding to $\left(C^{\prime}, \mathcal{E}^{\prime}\right) \in \mathcal{N}_{G}$ (resp. $\left(C_{1}, \mathcal{E}_{1}\right) \in$ $\left.\mathcal{N}_{M}\right)$, and assume that $E_{1}$ occurs in the restriction of $E$ to $\mathcal{W}_{1}$. Suppose that the theorem holds for $\sigma_{\left(C_{1}, \mathcal{E}_{1}\right)}$. If $X_{u, v} \neq \emptyset$ and $F$ acts trivially on $X_{u, v}$, then the theorem holds for $\sigma_{\left(C^{\prime}, \mathcal{E}^{\prime}\right)}$.

Proof. We follow the notation in Section 1. Since $E_{1}$ occurs in the restriction of $E$ to $\mathcal{W}_{1}, M_{E_{1}} \neq 0$ in (1.6.1). Since $\sigma_{\left(C^{\prime}, \mathcal{E}^{\prime}\right)}$ and $\sigma_{\left(C_{1}, \mathcal{E}_{1}\right)}$ is a scalar map, $\sigma_{\mathcal{E}_{1}, \mathcal{E}^{\prime}}$ is also a non-zero scalar map by (1.6.2). Since $F$ acts trivially on $X_{u, v}, \widetilde{\varepsilon}_{u, v}$ is the trivial extension of $\varepsilon_{u, v}$ to $\widetilde{A}(u, v)=\langle\tau\rangle \times A(u, v)$. It follows that

$$
\left\langle\widetilde{\varepsilon}_{u, v}, \widetilde{\rho \otimes \rho_{1}^{*}}\right\rangle_{A(u, v) \tau}=\left\langle\varepsilon_{u, v}, \rho \otimes \rho_{1}^{*}\right\rangle_{A(u, v)} .
$$

Then Corollary 1.9 together with Corollary 1.5 implies that

$$
\operatorname{Tr}\left(\sigma_{\mathcal{E}_{1}, \mathcal{E}^{\prime}}, M_{E_{1}}\right)=q^{-d_{C_{1}, C^{\prime}}+\operatorname{dim} U_{Q}} \operatorname{dim} M_{E_{1}},
$$

and we see that $\sigma_{\mathcal{E}_{1}, \mathcal{E}^{\prime}}$ is a scalar map by $q^{-d_{C_{1}, C^{\prime}}+\operatorname{dim} U_{Q}}$. By our assumption, $\sigma_{\left(C_{1}, \mathcal{E}_{1}\right)}$ is a scalar map by $q^{\left(a_{0}^{\prime}+r^{\prime}\right) / 2}$, where $a_{0}^{\prime}, r^{\prime}$ are as in 1.10 with respect to $M$. Thus again by (1.6.2), we see that $\sigma_{\left(C^{\prime}, \mathcal{E}^{\prime}\right)}$ is a scalar map by $q^{\left(a_{0}+r\right) / 2}$.

4.7. In view of Lemma 4.6, it is important to know the Frobenius action on $X_{u, v}$. We note that

(4.7.1) $Z_{G}(u) \times Z_{M}(v) U_{Q}$ acts transitively on the set $Y_{u, v}$.

In fact, put $\mathcal{Q}_{u, C_{1}}=\left\{g Q \in G / Q \mid g^{-1} u g \in C_{1} U_{Q}\right\} . \mathcal{Q}_{u, C_{1}}$ is a locally closed subvariety of $G / Q$. We have a surjective morphism $Y_{u, v} \rightarrow \mathcal{Q}_{u, C_{1}}$, $g \mapsto g Q$, which induces an isomorphism $Y_{u, v} / Z_{M}(v) U_{Q} \simeq \mathcal{Q}_{u, C_{1}}$. It is known by [Sp1, II, 6.7] that $Z_{G}(u)$ acts transitively on $\mathcal{Q}_{u, C_{1}}$. (4.7.1) follows from this. 
Lemma 4.8. If $Y_{u, v}^{F} \neq \emptyset$, then $F$ acts trivially on $X_{u, v}$.

Proof. Take $g \in Y_{u, v}$. By (4.7.1), the closure of $Z_{G}^{0}(u) g Z_{M}^{0}(v) U_{Q}$ in $Y_{u, v}$ gives an element $x \in X_{u, v}$, and $A_{G}(u) x A_{M}(v)$ gives all the irreducible components in $Y_{u, v}$. By assumption, we can choose $g \in Y_{u, v}^{F}$. Hence $Z_{G}^{0}(u) g Z_{M}^{0}(v) U_{Q}$ is $F$-stable, and so is $x$. Since $F$ acts trivially on $A_{G}(u)$ and $A_{M}(v), F$ stabilizes all the irreducible components in $Y_{u, v}$.

4.9. Assume that $F$ is of split type. For each $u \in G^{F}$, we shall find $v \in M^{F}$ such that $Y_{u, v}^{F} \neq \emptyset$. Let $V$ and $f$ be as in 2.1. Note that $G / Q$ can be identified with the subvariety of $\mathbf{P}(V)$ consisting of $\langle x\rangle$ for isotropic vectors $x$ with respect to $f,(\langle x\rangle$ denotes the line spanned by $x)$. Under the setting in $2.7,2.9$, we consider the vector space $\bar{V}=\bigoplus M_{j}$ which is identified with $V^{F}$. In the following cases, we can find $g \in G^{F}$ such that $g^{-1} u g \in Q$ and that $v=\pi\left(g^{-1} u g\right)$ is a split element in $M(\pi: Q \rightarrow M$ is the natural projection). In particular, we have $g \in Y_{u, v}^{F}$. In the discussion below, we identify the partitions with the corresponding Young diagrams.

First we consider the case where $G=S p_{2 n}$ or $S O_{N}$ with $p \neq 2$.

(i) Take $M_{j}$ such that $\varepsilon\left(\lambda_{j}\right)=1$. Let $e_{1}^{j}, \ldots, e_{h}^{j}$ be the basis of $M_{j}$ with $h=\lambda_{j}$ given in 2.9 (a). The stabilizer of $\left\langle e_{1}^{j}\right\rangle$ in $G$ is a parabolic subgroup $g Q g^{-1}$ with some $g \in G^{F}$. The nilpotent transformation $X_{j}$ on $M_{j}$ induces a map $\bar{X}_{j}$ on $\bar{M}_{j}=\left\langle e_{1}^{j}\right\rangle^{\perp} /\left\langle e_{1}^{j}\right\rangle$, and one can define a nilpotent element $\bar{X}$ on $\bar{M}_{j} \oplus \bigoplus_{j^{\prime} \neq j} M_{j^{\prime}}$. This determines a unipotent element $\pi\left(g^{-1} u g\right)=v$ in $M^{F}$ which is a split element. In this case $v$ is of type $\lambda^{\prime}$, where $\lambda^{\prime}$ is obtained from $\lambda$ by replacing one row $h$ such that $\varepsilon(h)=1$ by $h-2$.

(ii) Take $M_{j}$ such that $\varepsilon\left(\lambda_{j}\right)=1$ and that $c_{h}$ is even for $h=\lambda_{j}$. We choose $j$ such that $\lambda_{j}=\lambda_{j-1}=h$ and consider $N_{j}=M_{j} \oplus M_{j-1}$ with $f_{j}^{\prime}=f_{j}+f_{j-1}$. Thus $N_{j}$ has a basis $e_{1}^{j}, \ldots, e_{h}^{j}, e_{1}^{j-1}, \ldots, e_{h}^{j-1}$. By our construction, we have

$$
f_{j}\left(e_{1}^{j}, e_{h}^{j}\right)=-f_{j-1}\left(e_{1}^{j-1}, e_{h}^{j-1}\right)= \pm 1 .
$$

We consider $\bar{N}_{j}=\langle x\rangle^{\perp} /\langle x\rangle$ for $x=e_{1}^{j}+e_{1}^{j-1}$. The nilpotent transformation $X_{j}+X_{j-1}$ on $M_{j} \oplus M_{j-1}$ induces a linear map $\bar{X}_{j}$ on $\bar{N}_{j}$, and one can define a nilpotent element $\bar{X}$ on $\bar{N}_{j} \oplus \bigoplus M_{j^{\prime}}$. This determines a unipotent element $\pi\left(g^{-1} u g\right)=v$ in $M$ of type $\lambda^{\prime}$, where $\lambda^{\prime}$ is obtained from $\lambda$ by replacing two rows of length $h$ by two rows of length $h-1$. It is easy to see that $v$ is a split element in $M^{F}$. 
(iii) Take $M_{j}$ such that $\varepsilon\left(\lambda_{j}\right)=\omega$. Put $h=\lambda_{j}$. We choose a basis of $M_{j}$ as in 2.9 (b). Let $x=e_{1}^{j}+e_{1}^{j-1}$. Then $x$ is an isotropic vector with respect to $f_{j}$. Now $X_{j}$ induces $\bar{X}_{j}$ on $\bar{M}_{j}=\langle x\rangle^{\perp} /\langle x\rangle$, and one can define $\bar{X}$ on $\bar{M}_{j} \oplus \bigoplus_{j^{\prime} \neq j} M_{j^{\prime}}$. This determines a unipotent element $v=\pi\left(g^{-1} u g\right) \in M^{F}$. In this case, $v$ is of type $\lambda^{\prime}$, where $\lambda^{\prime}$ is obtained from $\lambda$ by replacing two rows of length $h$ by two rows of length $h-1$. It is easy to see that $v$ is a split element in $M^{F}$.

It is known (cf. [S1]) that if $Y_{u, v} \neq \emptyset$, for $u \in C_{\lambda}, v \in C_{\lambda^{\prime}}$, then $\lambda^{\prime}$ is obtained from $\lambda$ by the procedure described above. Hence if $v$ is split, it coincides with one of the above cases. It follows that

Proposition 4.10. Let $G=S p_{2 n}$ or $S O_{N}$ with $p \neq 2$. Assume that $F$ is of split type. Let $u \in G^{F}$ and $v \in M^{F}$ be such that $Y_{u, v} \neq \emptyset$. Assume that $u, v$ are split elements. Then $F$ acts trivially on $X_{u, v}$.

4.11. Next we consider the case where $G=S p_{2 n}$ with $p=2$. We keep the setting in 4.9 , in particular assume that $F$ is of split type.

(i) Choose $M_{j}$ as in 2.7 (a), i.e., the case where $\varepsilon(h)=1$ for $h=\lambda_{j}$. $v_{j}$ induces a linear map $\bar{v}_{j}$ on $\bar{M}_{j}=\left\langle e_{1}^{j}\right\rangle^{\perp} /\left\langle e_{1}^{j}\right\rangle$ and one can define $\bar{v}$ on $\bar{M}_{j} \oplus \bigoplus_{j^{\prime} \neq j} M_{j^{\prime}}$. This determines a unipotent element $\pi\left(g^{-1} u g\right)=v \in M$ for some $g \in G^{F} . v$ is contained in the class $C_{\lambda^{\prime}, \varepsilon^{\prime}}$ in $M$, where $\lambda^{\prime}$ is obtained from $\lambda$ by replacing one row of length $h$ by a row of length $h-2$, and $\varepsilon^{\prime}$ is given by $\varepsilon^{\prime}(h-2)=1$ and $\varepsilon^{\prime}\left(\lambda_{k}^{\prime}\right)=\varepsilon\left(\lambda_{k}\right)$ if $\lambda_{k}^{\prime} \neq h-2$. By our construction of the form $f_{j}$ in 2.5 , we see that $v$ is a split element if $h-2$ does not occur in the row of $\lambda$, nor $h-2$ occurs and $\varepsilon(h-2)=1$.

(ii) Assume that $c_{h} \geq 2$ and $\varepsilon(h)=1$ for $h=\lambda_{j}$, and take $\lambda_{j}=\lambda_{j-1}=$ $h$. We consider $M_{j}=V_{j} \oplus V_{j-1}$ with $f_{j}=f_{j}^{0}+f_{j-1}^{0}$, where $f_{j}^{0}, V_{j}$ are as in 2.7. Let $x=e_{1}^{j}+e_{1}^{j-1}$. Put $\bar{M}_{j}=\langle x\rangle^{\perp} /\langle x\rangle$. Then $v_{j}+v_{j-1}$ induces a unipotent element $\bar{v}_{j}$ on $\bar{M}_{j}$. This determines $\pi\left(g^{-1} u g\right)=v$ of $M^{F}$ as before. This construction is exactly the same as the one in 2.7 (b). Hence $v$ is contained in $C_{\lambda^{\prime}, \varepsilon^{\prime}}$ in $M$, where $\lambda^{\prime}$ is obtained from $\lambda$ by removing two rows of length $h$ by two rows of length $h-1$, and $\varepsilon^{\prime}(h-1)=\omega, \varepsilon^{\prime}\left(h^{\prime}\right)=\varepsilon\left(h^{\prime}\right)$ for all $h^{\prime} \neq h$. In this case, $v$ is a split element without any condition.

(iii) Choose $M_{j}$ as in 2.7 (b), i.e., the case where $\varepsilon(h)=\omega$. The basis of $M_{j}$ is given in 2.7 (b). Then $\bar{M}_{j}=\left\langle\bar{e}_{1}^{j}\right\rangle^{\perp} /\left\langle\bar{e}_{1}^{j}\right\rangle$ has a basis $\bar{e}_{n-1}^{j}, \bar{e}_{n-1}^{j-1}, \ldots, \bar{e}_{2}^{j}$, $\bar{e}_{2}^{j-1}$ (we use the same notation for the image on $\bar{M}_{j}$ as the one in $M_{j}$ ). Thus the induced linear map $\bar{v}_{j}$ on $\bar{M}_{j}$ is just a sum of two copies of nilpotent 
elements as given in 2.7 (a) with respect to the induced form $\bar{f}_{j}$ on $\bar{M}_{j}$. It follows that $\bar{v}$ determines a unipotent element $\pi\left(g^{-1} u g\right)=v \in M . v$ is contained in $C_{\lambda^{\prime}, \varepsilon^{\prime}}^{F}$, where $\lambda^{\prime}$ is obtained from $\lambda$ by replacing two rows of length $h$ by two rows of length $h-1$. $\varepsilon^{\prime}$ is given by $\varepsilon^{\prime}(h-1)=1$, and is the same as $\varepsilon$ for all other $h^{\prime} \neq h$. In particular, $v$ is a split element if $h-1$ does not occur in the rows of $\lambda$ nor $\varepsilon(h-1)=1$.

(iv) Choose $M_{j}$ as in (iii). Under the notation there, put $x=\bar{e}_{2}^{j}+\bar{e}_{2}^{j-1}$. Then $\bar{M}_{j}=\langle x\rangle^{\perp} /\langle x\rangle$ has a basis exactly the same as the basis of $N^{\perp} / N$ in 2.7 (c). Thus the induced map $\bar{v}_{j}$ on $\bar{M}_{j}$ determines a unipotent element $\pi\left(g^{-1} u g\right)=v$ of $M$ in the same way as above. $v$ is a split element in $M$ and is contained in $C_{\lambda^{\prime}, \varepsilon^{\prime}}$, where $\lambda^{\prime}$ is obtained from $\lambda$ by replacing two rows of length $h$ by two rows of length $h-1$, and $\varepsilon^{\prime}(h-1)=0$ if $h-1$ does not occur in $\lambda$ nor if $\varepsilon(h-1)=0$.

(v) Choose $M_{j}$ as in 2.7 (c), i.e., the case where $\varepsilon(h)=0$. The basis of $M_{j}$ is given in 2.7 (c). Then $\bar{M}_{j}=\left\langle\bar{e}_{1}^{j}\right\rangle^{\perp} /\left\langle\bar{e}_{1}^{j}\right\rangle$ has a basis $\bar{e}_{n-1}^{j}+$ $\bar{e}_{n-1}^{j-1}, \bar{e}_{n-2}^{j}, \bar{e}_{n-2}^{j-1}, \ldots, \bar{e}_{3}^{j}, \bar{e}_{3}^{j-1}, \bar{e}_{2}^{j}=\bar{e}_{2}^{j-1}$. Thus the induced linear map $\bar{v}_{j}$ on $\bar{M}_{j}$ is the same as the case (b) as above with respect to the induced form $\bar{f}_{j}$. It follows that $\bar{v}$ determines a unipotent element $\pi\left(g^{-1} u g\right)=v \in M . v$ is contained in $C_{\lambda^{\prime}, \varepsilon^{\prime}}$ where $\lambda^{\prime}$ is obtained from $\lambda$ by replacing two rows of length $h$ by two rows of length $h-1$, and $\varepsilon^{\prime}$ is given by $\varepsilon^{\prime}(h-1)=\omega$ if $h-1$ does not occur in the rows of $\lambda$. In this case, $v$ is a split element without any condition.

Finally, we consider the case where $G=S O_{2 n}$ with $p=2$. The argument in the case of $G=S p_{2 n}$ with $p=2$ works well for this case under a suitable modification, since in the discussion in Section 2, the induced quadratic form is of the same type as the original one, and so one can check easily that $v$ is a split element.

4.12. We consider the counter part of Proposition 4.10 and 4.11 for the case where $F=F_{0} \sigma$ is of non-split type. We assume that $G=S O_{2 n}(p$ : arbitrary) and $\widetilde{G}=O_{2 n}$. Let $u \in C_{\lambda, \varepsilon}^{F_{0}}$ and $v \in C_{\lambda^{\prime}, \varepsilon^{\prime}}^{F_{0}}$ be split elements as in 4.9 or 4.11, and assume that $Y_{u, v}^{F_{0}} \neq \emptyset$. Take $g \in Y_{u, v}^{F_{0}}$. Then $g^{-1} u g \in v U_{Q}$. By replacing $u$ by $g^{-1} u g$ (an element in the split class) we may assume that $u \in v U_{Q}$. Let $a=a_{i} \in Z_{\widetilde{G}}(u)$ be as in (2.10.1) and $\dot{a} \in Z_{\widetilde{G}}(u)$ be its representative. Let $a^{\prime} \in A_{\widetilde{M}}(v)$ be defined similar to $a$. We assume that $a$ and $a^{\prime}$ are both related to the row of the same length in $\lambda$ and $\lambda^{\prime}$. By the explicit description of the element $\dot{a} \in Z_{\widetilde{G}}(u)$ (see 2.7-2.10), we see that $\dot{a}$ normalizes $Q$. It follows that $\dot{a} \in Z_{\widetilde{M}}(v)$, which gives a representative of $a^{\prime}$. 
Let us write $\dot{a}=x s$ and let $\alpha^{-1} F(\alpha)=x$ be as in (2.10.1). Since we may take $s \in M$, we have $x \in M$, and so $\alpha \in M$ also. Let $u^{\prime}=\alpha u \alpha^{-1} \in C_{\lambda, \varepsilon}^{F}$ $v^{\prime}=\alpha v \alpha^{-1} \in C_{\lambda^{\prime}, \varepsilon^{\prime}}^{F}$ be split elements as given in (2.10.1). We have the following lemma.

Lemma 4.13. Let the notations be as above, and $u, v\left(\right.$ resp. $\left.u^{\prime}, v^{\prime}\right)$ be split elements with respect to $F_{0}$ (resp. F). Then under the assumption in Proposition 4.10 or $4.11, F$ acts trivially on the set $X_{u^{\prime}, v^{\prime}}$.

Proof. Since $\alpha \in M$, ad $\alpha$ maps $Y_{u, v}$ onto $Y_{u^{\prime}, v^{\prime}}$, and so induces a bijection between $X_{u, v}$ and $X_{u^{\prime}, v^{\prime}}$. Since $\dot{a} F_{0}=x F$, ad $\alpha$ maps $\dot{a} F_{0^{-} \text {-stable }}$ elements of $X_{u, v}$ to $F$-stable elements of $X_{u^{\prime}, v^{\prime}}$. In view of Proposition 4.10 and 4.11 , we may assume that $F_{0}$ acts trivially on the set $X_{u, v}$. Hence in order to prove the lemma, it is enough to show that any element in $X_{u, v}$ is stable by ad $\dot{a}$. Now an irreducible component of $Y_{u, v}$ is expressed as the closure of $Z_{G}^{0}(u) g Z_{M}^{0}(v) U_{Q}$ for some $g \in Y_{u, v}$. By our choice of $u$, $v$, we may take $g=1$. Then $Z_{G}^{0}(u) Z_{M}(v)^{0} U_{Q}$ is stable by ad $\dot{a}$, and so there exists an irreducible component stable by ad $\dot{a}$. Since $A_{G}(u) \times A_{M}(v)$ acts transitively on the set $X_{u, v}$, and $a$ commutes with $A_{G}(u)$ and $A_{M}(v)$, we conclude that ad $\dot{a}$ stabilizes each element in $X_{u, v}$. This proves the lemma.

4.14. In order to apply Lemma 4.6, we first need to know the condition for $X_{u, v} \neq \emptyset$. By the isomorphism $Y_{u, v} / Z_{M}(v) U_{Q} \simeq Q_{u, C_{1}}$ in 4.7, the elements in $X_{u, v}$ corresponds to the irreducible components of $Q_{u, C_{1}}$ of dimension $\left(\operatorname{dim} Z_{G}(u)-\operatorname{dim} Z_{M}(v)\right) / 2$. The condition for $C_{1}$ for the existence of such an irreducible component in $Q_{u, C_{1}}$ is described in [Sp1, II, 6.7]. By making use of 3.6, it is interpreted in terms of the symbols (cf. [LS, 2.6]);

(4.14.1) Let $\Lambda=\rho_{G}(u), \Lambda^{\prime}=\rho_{M}(v)$ be the distinguished symbols associated to $u$ and $v$. Then $X_{u, v} \neq \emptyset$ if and only if $\Lambda^{\prime}$ is obtained from $\Lambda$ by decreasing one of the entries of $\Lambda$ by 1 .

Let $E \in \mathcal{W}^{\wedge}, E_{1} \in \mathcal{W}_{1}^{\wedge}$ be as in Lemma 4.6. In applying the lemma, we also need to know when $E_{1}$ appears in the restriction of $E$. This is given as follows (cf. [LS, 2.8]).

(4.14.2) Let $X$ be the set of symbols as in 3.6. Let $(A, B) \in X$ corresponding to $E \in \mathcal{W}^{\wedge}$. Then $E_{1} \in \mathcal{W}_{1}^{\wedge}$ appears in the restriction of $E$ if and only if the symbol $\left(A^{\prime}, B^{\prime}\right)$ corresponding to $E_{1}$ is obtained from $(A, B)$ by decreasing one of the entries in $A$ or $B$ by 1. (This holds also for the case of degenerate 
symbols. If $E, E^{\prime} \in \mathcal{W}^{\wedge}$ are corresponding to the degenerate symbol $(A, A)$ and its copy, $E$ and $E^{\prime}$ have the same restriction on $\mathcal{W}_{1}$, and its components are parametrized by symbols obtained by decreasing one of the entries in $(A, A)$ by 1.) In particular, if $E_{1}$ appears in the restriction of $E$, we have $X_{u, v} \neq \emptyset$.

4.15. We are now ready to prove Theorem 4.2. So, assume that $F$ is of split type. First consider the case where $G=S p_{2 n}$ or $S O_{N}$ with $p \neq 2$. By induction on the rank of $G$, we may assume that the theorem holds for $S p_{2 n-2}$ or $S O_{N-2}$. Let $\left(C^{\prime}, \mathcal{E}^{\prime}\right) \in \mathcal{N}_{G}$ be corresponding to $E \in \mathcal{W}^{\wedge}$. Let $E_{1} \in \mathcal{W}_{1}^{\wedge}$ be an irreducible component of the restriction of $E$, and $\left(C_{1}, \mathcal{E}_{1}\right) \in \mathcal{N}_{M}$ be the corresponding element. We choose the split elements $u \in C^{\prime F}$ and $v \in C_{1}^{F}$. Then $X_{u, v} \neq \emptyset$ by (4.14.2), and $F$ acts trivially on $X_{u, v}$ by Proposition 4.10. Thus the assertion holds for $\sigma_{\left(C^{\prime}, \mathcal{E}^{\prime}\right)}$ by Lemma 4.6, and the theorem follows.

Next we consider the case where $G=S p_{2 n}$ or $S O_{2 n}$ with $p=2$. Since the result in 4.11 is somewhat weaker than Proposition 4.10, we need a more precise argument. Let $\lambda_{1} \leq \lambda_{2} \leq \cdots \leq \lambda_{2 m}$ be the sequence of $\lambda$ as in 3.6, and $\Lambda$ be the distinguished symbol associated to $\lambda$. For a given integer $h$, let $A_{h}=\left\{\lambda_{j}, \lambda_{j+1}, \ldots, \lambda_{k}\right\}$ be the subsequence consisting of $\lambda_{i}=h$. We say that $I=\{a, \ldots, b\}(a \leq b)$ is a semi-interval if $I$ corresponds to the sequence $A_{h}$ under the construction of $\Lambda$ in 3.6 (c), (d). The element $a$ is called the tail of the semi-interval $I$. They have the following forms. We denote by $I_{h}^{\varepsilon(h)}$ the semi-interval corresponding to $h$ and $\varepsilon(h)$.

$$
I_{h}^{\varepsilon(h)}= \begin{cases}\{a, a+2, a+4, a+6, \ldots\} & \text { if } \varepsilon(h)=1 \\ \{a, a, a+4, a+4, a+8, a+8, \ldots\} & \text { if } \varepsilon(h)=0 \\ \{a, a+1, a+4, a+5, a+8, a+9, \ldots\} & \text { if } \varepsilon(h)=\omega\end{cases}
$$

For two semi-intervals $I=\{a, \ldots, b\}, I^{\prime}=\left\{a^{\prime}, \ldots, b^{\prime}\right\}$ with $b^{\prime}<a$, the distance of $I, I^{\prime}$ is defined by $a-b^{\prime}$. It is easy to see that the distance of two semi-intervals is always $\geq 3$. The case where the distance is 3 occurs in the following three cases;

$$
\left(I_{h}^{1}, I_{h+2}^{1}\right), \quad\left(I_{h}^{1}, I_{h+1}^{\omega}\right), \quad\left(I_{h}^{\omega}, I_{h+1}^{1}\right) .
$$

The semi-interval $I$ is a part of an interval unless $I=I_{h}^{0}$, and if the distance of $I$ and $I^{\prime}$ is equal to 3, they are joined to be a part of one big interval. This explains the condition of generators of $A_{G}(u)$ in 2.4 . 
By induction, we may assume that the theorem holds for the smaller rank case. Let $E \in \mathcal{W}^{\wedge}$ corresponding to $\left(C^{\prime}, \mathcal{E}^{\prime}\right) \in \mathcal{N}_{G}$. Let $\Lambda$ be the distinguished symbol associated to $C^{\prime}$. We write $C^{\prime}=C_{\lambda, \varepsilon}$. Suppose that there exist two semi-intervals $I=\{a, \ldots, b\}, I^{\prime}=\left\{a^{\prime}, \ldots, b^{\prime}\right\}$ such that the distance $a-b^{\prime} \geq 5$. Then $a$ is also a tail of an interval, and it is easy to check that one can decrease $a$ by $a-1$ in $\Lambda$ to obtain a new symbol $\Lambda^{\prime}$. This procedure is also valid for a symbol similar to $\Lambda$. Moreover if $a$ corresponds to $h$, and $a-1$ corresponds to $h^{\prime}=h-1$ or $h-2$ under $C_{\lambda, \varepsilon} \leftrightarrow \Lambda$, then we have $c_{h^{\prime}}=0$. Let $C_{\lambda^{\prime}, \varepsilon^{\prime}} \leftrightarrow \Lambda^{\prime}$ with $v \in C_{\lambda^{\prime}, \varepsilon^{\prime}}^{F}$. There exists $\left(C_{1}, \mathcal{E}_{1}\right) \in \mathcal{N}_{M}\left(C_{1}=C_{\lambda^{\prime}, \varepsilon^{\prime}}\right)$ corresponding to $E_{1} \in \mathcal{W}_{1}^{\wedge}$ such that $E_{1}$ occurs in the restriction of $E$. By making use of 4.11, we see that $Y_{u, v}^{F} \neq \emptyset$ for a split element $v \in C_{\lambda^{\prime}, \varepsilon^{\prime}}$, and so $F$ acts trivially on $X_{u, v}$ by Lemma 4.8. Now Lemma 4.6 can be applied to show that the theorem holds for $\sigma_{\left(C^{\prime}, \mathcal{E}^{\prime}\right)}$.

Thus it is enough to consider the case where the distance of $I, I^{\prime}$ is $\leq 4$. Let $I=I_{h}^{\omega}$. There are three possibilities for $I^{\prime}$ with distance $\leq 4$, i.e., $I^{\prime}=I_{h-2}^{\omega}, I_{h-1}^{1}$ or $I_{h-1}^{0}$. For each case, one can find $\left(C_{1}, \mathcal{E}_{1}\right) \leftrightarrow E_{1}$ with a split element $v \in C_{1}^{F}=C_{\lambda^{\prime}, \varepsilon^{\prime}}^{F}$, to which Lemma 4.6 can be applied. For example consider the case where $I^{\prime}=I_{h-1}^{0}$. Then by (4.15.1),

$$
I^{\prime}=\{\ldots, a-4, a-4\}, \quad I=\{a, a+1, \ldots,\}
$$

for some $a$. It follows that $a$ is the tail of an interval. One can replace $I$ by $J=\{a, a, \ldots\}$ which produces a new symbol $\Lambda^{\prime}$ corresponding to $C_{\lambda^{\prime}, \varepsilon^{\prime}}$. This works also for a symbol $(A, B)$ similar to $\Lambda$, where $(A, B) \leftrightarrow E$. Now $\lambda^{\prime}$ is obtained from $\lambda$ by replacing two rows of length $h$ by two rows of length $h-1$. Since $\varepsilon(h-1)=0$, we have $\varepsilon^{\prime}(h-1)=0$ and 4.11 can be applied to show that $Y_{u, v}^{F} \neq \emptyset$ for a split element $v \in C_{\lambda^{\prime}, \varepsilon^{\prime}}^{F}$, and we get the assertion in a similar way as above. The other cases are dealt similarly.

Now we may assume that $I_{h}^{w}$ does not appear in $\Lambda$, i.e., assume that $\lambda$ consists of even rows. Assume that there exists $h$ such that $\varepsilon(h)=0$, and consider a semi-interval $I=I_{h}^{0}$. There are two possibilities for $I^{\prime}$ whose distance is $\leq 4$, i.e., $I^{\prime}=I_{h-2}^{1}$ or $I_{h-2}^{0}$. First assume that $I^{\prime}=I_{h-2}^{1}$. Then $I, I^{\prime}$ is written as

$$
I^{\prime}=\{\ldots, a-6, a-4\}, \quad I=\{a, a, a+4, a+4, \ldots\}
$$

by (4.15.1). Then one can replace the tail $a$ of $I$ by $a-1$, which divide $I$ into two semi-intervals

$$
J_{1}=\{a-1, a\}, \quad J_{2}=\{a+4, a+4, \ldots\},
$$


and $I^{\prime} \cup J_{1}$ form a part of some interval. The situation is the same for a symbol $(A, B)$ similar to $\Lambda$. This produces a new symbol $\Lambda^{\prime} \leftrightarrow C_{\lambda^{\prime}, \varepsilon^{\prime}}$ where $\lambda^{\prime}$ is obtained from $\lambda$ by replacing two rows of length $h$ by two rows of length $h-1$ where $\varepsilon^{\prime}(h-1)=\omega$. Hence by 4.11 , we have $Y_{u, v}^{F} \neq \emptyset$ for a split element $v \in C_{\lambda^{\prime}, \varepsilon^{\prime}}$. Another case is dealt similarly.

Finally we may assume that $\lambda$ consists of even rows $h$ with $\varepsilon(h)=1$. We consider $I=I_{h}^{1}, I^{\prime}=I_{h-2}^{1}$. Then we have

$$
I^{\prime}=\{\ldots, a-5, a-3\}, \quad I=\{a, a+2, a+4, \ldots\} .
$$

Note that $I, I^{\prime}$ are a part of a common interval. By replacing $a$ by $a-1$, we have new semi-intervals

$$
J^{\prime}=\{\ldots, a-5, a-3, a-1\}, \quad J=\{a+2, a+4, \ldots\} .
$$

This produces a new symbol $\Lambda^{\prime} \leftrightarrow C_{\lambda^{\prime}, \varepsilon^{\prime}}$, where $\lambda^{\prime}$ is obtained from $\lambda$ by replacing one row of length $h$ by one row of length $h-2$. Since $\varepsilon(h-2)=1$, the argument in 4.11 can be applied, and we get the assertion of the theorem. Theorem 4.2 is now proved.

4.16. We shall prove Theorem 4.3. So assume that $F$ is of non-split type. First consider the case where $L \neq T$. In this case the proof is done almost similar to the proof of Theorem 4.2 , by using Lemma 4.13 instead of Proposition 4.10 and 4.11. However, we have to be careful for the choice of $v$ (cf. the condition of $a$ and $a^{\prime}$ in 4.12) in applying Lemma 4.13. In the case where $p=2$, this is done along the line in 4.15 , by choosing the decreasing number suitably. In the case where $p \neq 2$, Proposition 4.10 cannot be applied directly, and we have to apply a similar argument as in 4.15. But this is easier than the case of $p=2$; the semi-intervals are of the form $I_{h}^{1}=\{a, a+1, a+2, \ldots\}$ or $I_{h}^{\omega}=\{a, a, a+2, a+2, \ldots\}$, and only $I_{h}^{1}$ gives an interval. The distance of two semi-intervals $I, I^{\prime}$ is $\geq 2$. If the distance is $\geq 3$, no interaction occurs for $I, I^{\prime}$ in decreasing one entry. Then the assertion (i) of the theorem is obtained by considering the following $\left(I, I^{\prime}\right)$.

$$
\left(I_{h}^{\omega}, I_{h-2}^{\omega}\right), \quad\left(I_{h}^{\omega}, I_{h-1}^{1}\right), \quad\left(I_{h}^{1}, I_{h-1}^{\omega}\right), \quad\left(I_{h}^{1}, I_{h-2}^{1}\right) .
$$

The details are omitted.

Next we consider the case where $L=T$. Hence $\mathcal{W}=W_{n}^{\prime}$, and we regard it as a subgroup of $W_{n}=W_{n}^{\prime}\langle\sigma\rangle$. We consider the decomposition of $E=V_{\left(C^{\prime}, \mathcal{E}^{\prime}\right)}$ in (1.6.1). Assume that $E$ is $F$-stable, and let $\widetilde{E}$ be the 
extension of $E$ by $\sigma_{\left(C^{\prime}, \mathcal{E}^{\prime}\right)}$. Let $\widetilde{E}_{1}$ be the extension of $E_{1}=V_{\left(C_{1}, \mathcal{E}_{1}\right)}$ through $\sigma_{\left(C_{1}, \mathcal{E}_{1}\right)}$. Note that in our case $\operatorname{dim} M_{E_{1}}=1$, and so $\sigma_{\mathcal{E}^{\prime}, \mathcal{E}}$ is a scalar map. By multiplying $\sigma^{-1}$ on the both side of (1.6.2), one can write

$$
\left.\sigma^{-1} \circ \sigma_{\left(C^{\prime}, \mathcal{E}^{\prime}\right)}\right|_{M_{E_{1}} \otimes E_{1}}=\sigma_{\mathcal{E}^{\prime}, \mathcal{E}_{1}} \otimes \sigma^{-1} \circ \sigma_{\left(C_{1}, \mathcal{E}_{1}\right)} .
$$

Since $F$ acts trivially on $A_{G}(u)$ and $A_{M}(v)$, the extension $\widetilde{\rho \otimes \rho_{1}^{*}}$ is the trivial extension. Thus if $F$ acts trivially on $X_{u^{\prime}, v^{\prime}}$, then Corollary 1.9 implies that $\sigma_{\mathcal{E}^{\prime}, \mathcal{E}_{1}}$ is a scalar map by $q^{-d_{C_{1}, C^{\prime}}+\operatorname{dim} U_{Q}}$. Now assume that $E$ corresponds to the symbol $\Lambda$ and $E_{1}$ is an irreducible component of $E$ corresponding to the symbol $\Lambda^{\prime}$, where $\Lambda^{\prime}$ is obtained from $\Lambda$ by decreasing an entry by 1 , under the condition in 4.12 . (Note that $\Lambda$ is not a degenerate symbol since $E$ is $F$-stable.) Let $\widetilde{E}$ and $\widetilde{E}_{1}$ be the preferred extensions of $E, E_{1}$, respectively. Then it is easy to check that $\widetilde{E}_{1}$ occurs in the restriction of $\widetilde{E}$ on $W_{n-1}$. Thus again by using the arguments in 4.15 (see also the remark for the case where $L \neq T$ with non-split case), thanks to Lemma 4.13, the verification of Theorem 4.3 (ii) is reduced to the case where $n=2$, namely $G \simeq G_{1} \times G_{1}$, where $G_{1}$ is of type $A_{1}$, and $F$ acts as a permutation of two factors. This case is checked as follows (cf. [S1, Lemma 3.11]). Since the class $C^{\prime}$ is $F$-stable, $u^{\prime}$ is the product of two regular elements in $G_{1}$ or the product of two identity elements in $G_{1}$. Let $\mathcal{B}_{u^{\prime}}$ be the variety of Borel subgroups of $G$ containing $u^{\prime}$. Then $F$ acts trivially on the one dimensional $W_{n}^{\prime}$-module $H^{2 d_{u^{\prime}}}\left(\mathcal{B}_{u^{\prime}}\right)$, where $d_{u^{\prime}}=\operatorname{dim} \mathcal{B}_{u^{\prime}}$. Hence $W_{n}$-module $H^{2 d_{u^{\prime}}}\left(\mathcal{B}_{u^{\prime}}\right)$ coincides with the identity representation or the long sign representation $\eta$ (i.e., takes the value $\eta\left(r_{\alpha}\right)=1, \eta\left(r_{\beta}\right)=-1$, where $r_{\alpha}$ (resp. $r_{\beta}$ ) is the reflection with respect to the short root $\alpha$ (resp. long root $\beta$ ) of the root system of type $B_{2}$.) according to the cases where $u^{\prime}$ is regular or identity. The corresponding symbol is $(2 ; 0)$ for the former, and $(12 ; 01)$ for the latter. The both are preferred extensions, and the theorem follows.

\section{REFERENCES}

[D] J. Dieudonné, La Géométrie des Groupes Classiques, Springer-Verlag, 1971.

[L1] G. Lusztig, Intersection cohomology complexes on a reductive group, Invent. Math., 75 (1984), 205-272.

[L2] G. Lusztig, Character sheaves, I, Adv. in Math., 56 (1985), 193-237, II, Adv. in Math., 57 (1985), 226-265, III, Adv. in Math., 57 (1985), 266-315, IV, Adv. in Math., 59 (1986), 1-63, V, Adv. in Math., 61 (1986), 103-155.

[LS] G. Lusztig and N. Spaltenstein, On the generalized Springer correspondence for classical groups, Advanced Studies in Pure Math. Vol. 6 (1985), pp. 289-316. 
[S1] T. Shoji, On the Green polynomials of classical groups, Invent. Math., 74 (1983), 237-267.

[S2] T. Shoji, Generalized Green functions and unipotent classes for finite reductive groups, I, Nagoya Math. J., 184 (2006), 155-198.

[Sp1] N. Spaltenstein, Classes Unipotentes et Sous-groupes de Borel, Lecture Note in Math. 946, Springer-Verlag, 1982.

[Sp2] N. Spaltenstein, On the generalized Springer correspondence for exceptional groups, Advanced Studies in Pure Math. Vol. 6 (1985), pp. 317-338.

[SS] T. A. Springer and R. Steinberg, Conjugacy classes, Seminar on Algebraic Groups and Related Finite Groups, Lect. Note in Math. 131, Springer-Verlag (1970), pp. 167-266.

Graduate School of Mathematics

Nagoya University

Chikusa-ku

Nagoya, 464-8602

Japan 